\title{
Epitope-based chimeric peptide vaccine design against $S, M$ and E proteins of SARS-CoV-2 etiologic agent of global pandemic COVID-19: an in silico approach
}

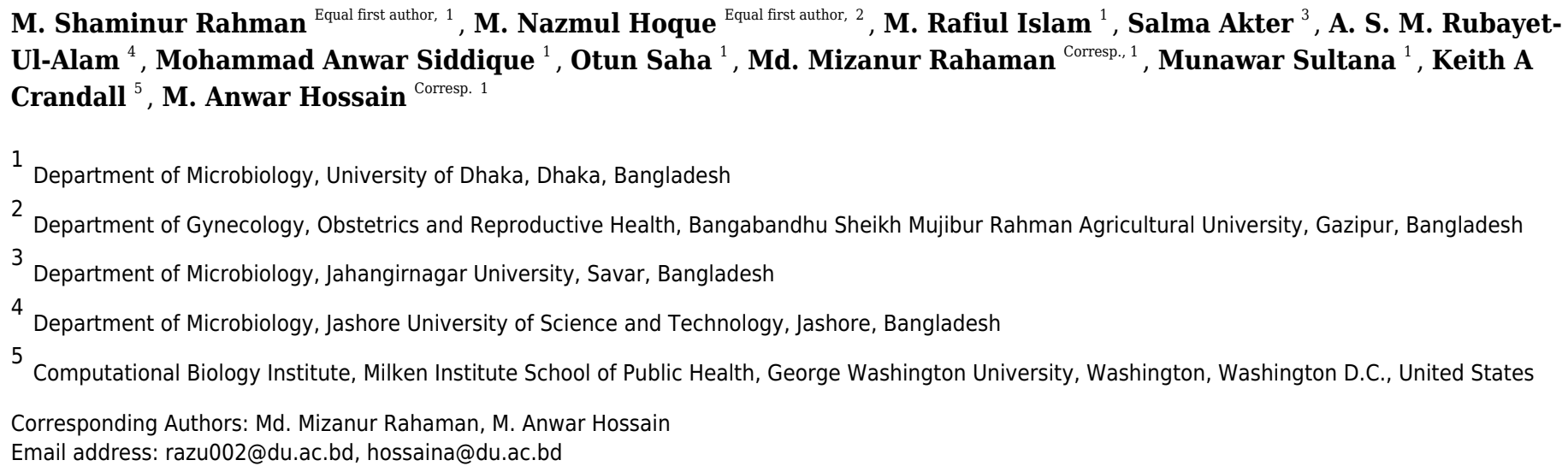

Severe acute respiratory syndrome coronavirus 2 (SARS-CoV-2) is the cause of the ongoing pandemic of coronavirus disease 2019 (COVID-19), a public health emergency of international concern declared by the World Health Organization (WHO). An immunoinformatics approach along with comparative genomic was applied to design a multiepitope-based peptide vaccine against SARS-CoV-2 combining the antigenic epitopes of the $S, M$ and $E$ proteins. The tertiary structure was predicted, refined and validated using advanced bioinformatics tools. The candidate vaccine showed an average of $\geq 90.0 \%$ world population coverage for different ethnic groups. Molecular docking and dynamics simulation of the chimeric vaccine peptide with the immune receptors (TLR3 and TLR4) predicted efficient binding. Immune simulation predicted significant primary immune response with increased IgM and secondary immune response with high levels of both IgG1 and IgG2. It also increased the proliferation of T-helper cells and cytotoxic T-cells along with the increased INF-y and IL-2 cytokines. The codon optimization and mRNA secondary structure prediction revealed the chimera is suitable for high-level expression and cloning. Overall, the constructed recombinant chimeric vaccine candidate demonstrated significant potential and can be considered for clinical validation to fight against this global threat, COVID-19. 
1 Epitope-based chimeric peptide vaccine design against S, M and E proteins of SARS-CoV-2

2

3

4 M. Shaminur Rahman ${ }^{*}$, M. Nazmul Hoque ${ }^{1,2 *}$, M. Rafiul Islam ${ }^{1}$, Salma Akter ${ }^{1,3}$, A. S. M.

5 Rubayet-Ul-Alam ${ }^{1,4}$, Mohammad Anwar Siddique ${ }^{1}$, Otun Saha ${ }^{1}$, Md. Mizanur Rahaman ${ }^{\text {*** }}$,

6 Munawar Sultana ${ }^{1}$, Keith A. Crandall ${ }^{5}$, M. Anwar Hossain ${ }^{1,6^{* *}}$

$7 \quad{ }^{1}$ Department of Microbiology, University of Dhaka, Dhaka-1000, Bangladesh

8 2Department of Gynecology, Obstetrics and Reproductive Health, Bangabandhu Sheikh Mujibur

9 Rahman Agricultural University, Gazipur-1706, Bangladesh

$10{ }^{3}$ Department of Microbiology, Jahangirnagar University, Savar, Dhaka-1342, Bangladesh

$11{ }^{4}$ Department of Microbiology, Jashore University of Science and Technology, Jashore-7408,

12 Bangladesh

$13{ }^{5}$ Computational Biology Institute, Milken Institute School of Public Health, The George

14 Washington University, USA

15 'Present address: Jashore University of Science and Technology, Jashore-7408, Bangladesh

$16 *$ Equal contribution

$17 * *$ Corresponding to:

18

M. Anwar Hossain, PhD

Professor

Department of Microbiology

University of Dhaka, Dhaka, Bangladesh

E-mail: hossaina@du.ac.bd

Or,

Dr. Md. Mizanur Rahaman

Assistant Professor

Department of Microbiology

University of Dhaka, Dhaka, Bangladesh

E-mail: razu002@du.ac.bd 


\section{Abstract}

32

51 Keywords: SARS-CoV-2, Muti-epitope, Chimeric Peptide Vaccine, B-cell Epitope, T-cell

Severe acute respiratory syndrome coronavirus 2 (SARS-CoV-2) is the cause of the ongoing pandemic of coronavirus disease 2019 (COVID-19), a public health emergency of international concern declared by the World Health Organization (WHO). An immuno-informatics approach along with comparative genomic was applied to design a multi-epitope-based peptide vaccine against SARS-CoV-2 combining the antigenic epitopes of the $\mathrm{S}, \mathrm{M}$ and $\mathrm{E}$ proteins. The tertiary structure was predicted, refined and validated using advanced bioinformatics tools. The candidate vaccine showed an average of $\geq 90.0 \%$ world population coverage for different ethnic groups. Molecular docking and dynamics simulation of the chimeric vaccine peptide with the immune receptors (TLR3 and TLR4) predicted efficient binding. Immune simulation predicted significant primary immune response with increased $\operatorname{IgM}$ and secondary immune response with high levels of both IgG1 and IgG2. It also increased the proliferation of T-helper cells and cytotoxic T-cells along with the increased INF- $\gamma$ and IL-2 cytokines. The codon optimization and mRNA secondary structure prediction revealed the chimera is suitable for high-level expression and cloning. Overall, the constructed recombinant chimeric vaccine candidate demonstrated significant potential and can be considered for clinical validation to fight against this global threat, COVID-19. 


\section{Introduction}

54

55

56

57

58

59

60

61

62

63

64

65

66

67

68

Emergence of the SARS-CoV-2, which was first reported in Hubei Province of Wuhan, China in December 2019, is responsible for the ongoing global pandemic of coronavirus disease 2019 (COVID-19) spreading across 216 countries, areas or territories with 6,992,274 active infection cases and 403,128 deaths until June 9 $9^{\text {th }}, 2020$ (WHO, 2020). This SARS-CoV-2 is the third coronovairus $(\mathrm{CoV})$ belonging to Genus Betacoronavirus that can infect human after the two previously reported coronavirus- severe acute respiratory syndrome (SARS-CoV) (Almofti et al., 2018; Wu et al., 2020), and Middle East respiratory syndrome (MERS-CoV) (Badawi et al., 2016; Pallesen et al., 2017; ul Qamar et al., 2019). The SARS-CoV-2 has a positive-sense, singlestranded, and $\sim 30$ kilobase long RNA genome showing $79.0 \%$ and $50.0 \%$ identity to the genomes of SARS-CoV and MERS-CoV, respectively (Abdelmageed et al., 2020; Tang et al., 2020; Lu et al., 2020; Hoque et al., 2020a). Among multiple encoded proteins (structural, non-structural and accessory), four major structural proteins are the spike (S) glycoprotein, small envelope protein (E), membrane protein (M), and nucleocapsid protein (N) (Ahmed et al., 2020).

The S glycoprotein, because of its higher antigenicity and surface exposure (Almofti et al., 2018; Zhou et al., 2019; Shang et al., 2020), plays the most crucial role for the attachment and entry of viral particles into the host cells through the host angiotensin-converting enzyme 2 (ACE2) receptor (Gralinski et al., 2020; Shang et al., 2020). It is noteworthy that $\mathrm{E}$ and M proteins also have important functions in the viral assembly, budding and replication of virus particles, as well as play role in augmenting the immune response against SARS-CoV (Shi et al., 2006; Schoeman \& Fielding, 2019; Shang et al., 2020). Monoclonal antibodies (mAbs) primarily target the trimeric S glycoprotein of the virion consisting of three homologous chains (A, B and C), and this protein is composed of two major domains, the receptor-binding domain (RBD) and the N- 
76 terminal domain (NTD) (Pallesen et al., 2017; Song et al., 2018; Zhou et al., 2019; Wrapp et al.,

77 2020). The NTD is located on the side of the spike trimer and has not been observed to undergo

78 any dynamic conformational changes (Shang et al., 2020). Thus, this specific region might play

79 role in viral attachment, inducing neutralizing antibody responses and stimulating a protective

80 cellular immunity (Almofti et al., 2018; ul Qamar et al., 2019; Shang et al., 2020).

Most of the recent vaccine candidates induce neutralizing antibodies against the different forms and/or variants of the spike protein of the SARS-CoV-2 (Le et al. 2020). However, the immune responses generated from using single protein have generally been inadequate to warrant their use in the development of an effective prophylactic tool (Shi et al., 2015; Shey et al., 2019). On this note, multi-epitope vaccine candidates have already been designed against several viruses, including MERS-CoV and SARS-CoV, and their efficacies have been further reported (Almofti et al., 2018; ul Qamar et al., 2019; Yong et al., 2019). Two related studies have reported the in-silico design of epitope based chimeric vaccine candidates targeting E, M, S and N proteins of SARSCoV-2, albeit not peer-reviewed (Yazdani et al., 2020; Akhand et al., 2020). Additionally, Kibria et al. (2020) performed an immunoinformatic approach to design a 70 aa long multi-epitope vaccine focusing on the the virion outer surface proteins (E, M, and S) (Chan et al., 2020). COVID-19 based on the genomics, functional structures, and host-pathogen interactions, however the ultimate results of these efforts is yet uncertain. Currently, 10 candidate vaccines against SARS-CoV-2 are in the clinical trial, and 121 more under preclinical evaluation (WHO, 2020). Strikingly, researchers are trialing different technologies, albeit targeting spike protein mainly, some of which have not been used in a licensed vaccine before (Le et al., 2020). Their vaccine appears to be effective and safe based on a limited data and application of the vaccine within a 
99 relatively tiny group of individuals. Nevertheless, there are several uncertainties, for example,

100 whether it will relate to antibody responses in the general population, be safe within a specific sub-

101 population (children, pregnant women, and elder people), as well as lack of a standardized virus

102 neutralization assay and accurate vaccine titer are complicating data interpretation. Moreover, only

103 half of the medium dose receiver developed neutralizing antibody and the T-cell response is not

104 particularly impressive (Sheridan, 2020).

105

Thus, mimicking of a more natural state of the virus where surface exposed proteins, or the

106 immunodominant epitopes of those proteins influence the immune response might be a solution,

107 if those candidates ultimately cannot meet the final goal. Furthermore, excluding the nucleocapsid

108 (N) protein, embedded within the structure and attached to the viral genome, will amplify the 109 chance of developing a more pseudo-virus state for the expressed chimera that may produce 110 specific antibody as well as T-cell responses. Besides, peptide-based chimeric vaccines are 111 biologically safe as they do not need in vitro virus culture, and their selectivity might ensure 112 accurate activation of specific immune responses (Dudek et al., 2010; Wang et al., 2019).

113 Considering the facts, we have proposed the development of a multi-epitope vaccine candidate, 114 which differs from all the previous studies in the aspect of containing whole RBD and NTD regions 115 of the spike protein alongwith specific epitopes of $M$ and E proteins giving an excellent chimeric 116 conformation and might lead to the generation of a more potent protective immune response since 117 smaller epitopes have less ability to give better immune protection. Hence, we can assume that 118 chimeric vaccine targeting multiple epitopes on the RBD and NTD segments of the S protein, M 119 and E proteins would be a potentially effective vaccine candidate in combatting SARS-CoV-2, 120 and therefore, could be used against the globally pandemic highly pathogenic SARS-CoV-2. 


\section{Results}

122 Comparative structural analysis of SARS-CoV, MERS-CoV and SARS-CoV-2

124 and 31.93\% sequence identity with the S proteins of the SARS-CoV and MERS-CoV, respectively

125 (Fig. S1). The structural (validated using the Ramachandran plot as in Fig. S2) alignment of the

126 coronavirus S proteins reflects high degree of structural heterogeneity in the receptor-binding 127 domain (RBD) and N-terminal domain (NTD) of the chain A and chain C compared to that of 128 chain B (Fig. S3). Divergence of individual structural domains, NTD and RBD of 2019-nCoV 129 spike protein from both of the SARS-CoV and MERS-CoV warrants the domains for epitope130 based chimeric vaccine development, particularly against SARS-CoV-2.

\section{Screening for B-cell epitopes}

Linear epitopes prediction (ElliPro) based on solvent-accessibility and flexibility revealed the potential epitope candidate because of their presence in viral transmembrane domains (Fig. S4). The tertiary structures of the RBD and NTD illustrate their surface-exposed position on the S protein (Fig. 1). Using IEDB analysis resource and Bepipred linear epitope prediction 2.0 tools,

141 protein, while the $\mathrm{E}$ and $\mathrm{M}$ proteins had 2 and 6 epitopes, respectively (Fig. 2, Table S1). However, 
142 only 5 epitopes were exposed on the surface of the virion, and had a high antigenicity score (>

143 0.4), indicating their potentials in initiating immune responses (Table 2).

144 Among the five annotated epitopes having antigenicity score of $\geq 0.5$ (VaxiJen 2.0 tool),

145 RBD and NTD regions each possessed two highly antigenic epitopes while the envelope (E)

146 protein contained only one highly antigenic epitope and membrane $(\mathrm{M})$ protein has none (Table

147 2). Furthermore, the Kolaskar and Tongaonkar antigenicity profiling found five highly antigenic

148 epitopes in RBD region with an average (antigenicity) score of 1.042 (minimum $=0.907$,

149 maximum $=1.214$ ), and seven highly antigenic epitopes in NTD with an average (antigenicity)

150 score of $1.023($ minimum $=0.866$, maximum $=1.213)($ Fig. S5, Table S2). The average Kolaskar

151 scores for envelope protein B-cell epitope (EBE) and membrane protein B-cell epitope (MBE)

152 were 0.980 and 1.032, respectively (Table S2). However, through ABCPred analysis, we further

153 verified 18 and 11 B-cell epitopes in RBD and NTD regions with average antigenicity score of

1540.775 and 0.773 in the associated domains, respectively (Table S3).

\section{Selection of T-cell and IFN- $\gamma$ inducing epitopes}

The IEDB MHC-I prediction tool retrieved 77 T-cell epitopes in RBD that interacted with

15721 possible MHC-I alleles whereas the NTD domain possessed 35 T-cell epitopes with 17 possible

158 MHC-I alleles (Supplemental Data 1). Similarly, the IEDB MHC-II prediction tool generated 13-

159 mer 124 peptides from the RBD, and 10-mer 73 peptides in the NTD segments of the S protein

160 that showed interaction with many different and/or common MHC-II alleles with an $\mathrm{IC}_{50}$ value

161 ranging from 1.4 to $49.9 \mathrm{nM}$ (Supplemental Data 1). Furthermore, the analysis tool of the IEDB

162 generated an overall scores for proteasomal processing, TAP transport, and MHC-binding 
163 efficiency indicating the intrinsic potential of the epitopes to be recognized by immunoreactive T-

164 cells (Supplemental Data 1). of S protein, and membrane protein B-cell linear epitope (MBE) had great probability to release of IFN- $\gamma$ with a positive score. A total of 56 potential positive IFN- $\gamma$ inducing epitopes (15-mer) were predicted for the RBD domain with an average epitope prediction score of 0.255 and the maximum SVM score of 0.625 . On the other hand, a total of 33 potential positive epitopes were predicted for the NTD domain with an average epitope prediction score of 0.312 and the maximum SVM score of 0.811 . Moreover, the M protein also possessed several IFN- $\gamma$ inducing epitopes having an average epitope prediction score of 0.980 (Table S4).

Design-construction, antigenicity and physiochemical properties of the chimeric vaccine candidate MBE (20 aa), NTD (139 aa), RBD (200 aa), EBE (15 aa), and Invasin (16 aa), and the construct was named as CoV-RMEN (417 aa) as shown in Fig. 3a. These segments were connected with a repeat of hydrophobic (glycine; G) and acidic aa (glutamic acid; E) linkers for making the final vaccine construct more flexibile with balanced ratio of acidic and basic amino acids. The molecular weight (MW) of the CoV-RMEN was $46.8 \mathrm{kDa}$ with a predicted isoelectric point (pI) of 8.71. The projected half-life was 4.4 hours in mammalian reticulocytes in vitro, and $>20$ hours in yeast and $>10$ hours in E. coli in vivo. The protein was predicted to be less soluble upon expression with a solubility score of 0.330 . An instability index (II) value 29.74 predicted the protein as stable (II of $184>40$ indicates instability). The estimated aliphatic index was 66.59 , indicating thermostability of 
185 the final chimera. The predicted grand average of hydropathicity (GRAVY) was -0.300 . The

186 antigenicity score of 0.450 was predicted by the VaxiJen 2.0 server with a virus model at a 187 threshold of 0.4 , and further verified by ANTIGENpro showing score of 0.875 (maximum 188 expected score ranking is 1.0) indicating the high antigenic nature of the designed vaccine, CoV189 RMEN . Moreover, the vaccine was also predicted to be non-allergenic on both the AllerTOP v. 2 190 and AllergenFP servers.

191

192

193

194

195 196 197 198 199 200 201 202 203 204 205 206

\section{Structural characterization of the CoV-RMEN}

The CoV-RMEN peptide was predicted to contain $43.2 \%$ alpha helix, $67.4 \%$ beta sheet, and 12\% turns (Fig. 3b, Fig. S6) using CFSSP:Chou and Fasman secondary structure prediction server. Additionally, regarding the solvent accessibility of aa residues, 34\%, 30\% and 34\% were predicted to be exposed, medium exposed and buried respectively (Fig. S7). The RaptorX Property server predicted only two aa residues in the disordered domains. The Phyre 2 server predicted the tertiary structure model of the designed chimeric protein in 5 templates $(\mathrm{c} 5 \mathrm{x} 5 \mathrm{bB}, \mathrm{c} 2 \mathrm{~mm} 4 \mathrm{~A}$, $\mathrm{c} 6 \mathrm{vsbB}, \mathrm{c} 5 \mathrm{x} 29 \mathrm{~B}$ and $\mathrm{c} 6 \mathrm{vybB})$ based opon heuristics to maximize confidence, alignment coverage and percent identity. The final 3D structure of the CoV-RMEN peptide modelled at $82 \%$ with more than $90 \%$ confidence (Fig. 3c). Moreover, 65 residues were modelled by ab initio. The selected structural model has parameters of RMSD (0.414), GDT-HA (0.9538), and MolProbity (2.035).

The Ramachandran plot analysis of the finally modelled protein exhibited $94.7 \%$ of the aa residues in favored regions (Fig. 3d), consistent with the $94.0 \%$ score predicted by the GalaxyRefine analysis. Additionally, $4.8 \%$ of the residues located in allowed regions, and only $0.5 \%$ in disallowed regions (Fig. 3d). The chosen model after refinement had an overall quality factor of 74.45\% with ERRAT (Fig. S8) and a ProSA-web based Z-score of - 6.17(Fig. 3e). 
207 Molecular docking and dynamics simulation analysis

208

209

210

211

212

213

214

215

216

217

218

219

220

221

222

223

224

225

226

227

228

229

Among the selected epitopes from the RBD and NTD segments, top five based on $\mathrm{IC}_{50}$ score (Table 2) revealed highly favorable molecular interaction for stable binding with their respective HLA alleles. Docking complexes thus formed have significantly negative binding affinity $\left(\Delta \mathrm{G}\right.$ always remained $\leq-8.2 \mathrm{kcal} \mathrm{mol}^{-1}$, average $\left.=-9.94 \mathrm{kcal} \mathrm{mol}^{-1}\right)$, and most of the amino acid (aa) residues of the epitopes were involved in molecular interactions with their respective HLA alleles (Fig. 4, Supplemental data 1). The immune responses of TLR2, TLR3 and TLR4 against vaccine construct (CoV-RMEN) were estimated by analyzing the overall conformational stability of vaccine protein-TLRs docked complexes. The active interface aa residues of refined complexes of CoV-RMEN and TLRs were predicted (Fig. 5, Table 3).

The relative binding free energies $(\Delta \mathrm{G})$ of the protein-TLRs complexes were significantly negative (Table 3) which suggest that the interaction of the chimeric protein might favor stimulation of the TLR receptors. Consistently, the number of contacts made at the interface (IC) per property (ICs charged-charged: 5, ICs charged-polar: 2, ICs charged-apolar: 17, polar-polar: 1, ICs polar-apolar: 7 and apolar-apolar: 16) for the vaccine protein-TLR2 complex. Interface contacts (IC) per property (ICs charged-charged: 16, ICs charged-polar: 22, ICs charged-apolar: 26, polar-polar: 6, ICs polar-apolar: 25 and apolar-apolar: 29) were for the vaccine protein-TLR3 complex. Also, vaccine protein-TLR4 complex showed similar (ICs) per property (ICs chargedcharged: 5, ICs charged-polar: 11, ICs charged-apolar: 30, polar-polar: 4, ICs polar-apolar: 31 and apolar-apolar: 39). Furthermore, the molecular dynamics (MD) simulation analysis of the docked CoV-RMEN-TLR3 and CoV-RMEN-TLR4 complexes showed soundly stable RMSD values between $\sim 4.35$ and $\sim 5.4 \mathrm{~nm}$ for a specified time frame of $100 \mathrm{ps}$ at the reasonably consistent temperature $(\sim 300 \mathrm{~K})$ and pressure (1bar), whereas CoV-RMEN-TLR2 complex showed RMSD 
230 value between 5.5 and 6.2 with same cut-off parameters. These data validated that the docked

231 complexes (CoV-RMEN-TLR3 and CoV-RMEN -TLR4) are more stable than CoV-RMEN-TLR2

232 (Fig. 5).

233 Immune simulation

234

The cumulative results of immune responses after three times antigen exposure with four-

235 week interval each time revealed that the primary immune response against the antigenic fragments

236 was elevated indicated by gradual increase of IgM level after each antigen exposure (Fig. 6a).

237 Besides, the secondary immune response, crucial for immune stability, have been shown as

238 increased with adequate generation of both $\operatorname{IgG} 1$ and $\mathrm{IgG} 2$. Also, the elevated level of all

239 circulating immunoglobulins indicates the accuracy of relevant clonal proliferation of B-cell and

240 T-cell population. The level of cytokines after antigen exposure increased concomitantly reflected

241 by escalation of IFN- $\gamma$ and IL-2, which are most significant cytokines for anti-viral immune

242 response and clonal selection (Fig. 6b). The abundance of different types of B-cells and T-cells,

243 like antigen processing B-cells, resting memory B- and T- cells, B-cells with IgM and IgG remains

244 significantly higher indicating development of immune memory and consequently increased

245 clearance of antigen after exposure (Fig. 6c,d). Additionally, T-helper cells and cytotoxic T-cells

246 were found with a drastic up-regulation of Th1 concentration enhancing the B-cell proliferation

247 and immune memory development (Fig. 6e,f). The high level of immunoglobulin IgG1 + $\operatorname{IgG} 2$,

248 active B-cell and T-helper cell population reflected the development of strong immune response

249 reinforcing the indelible and peerless antigenicity of the CoV-RMEN vaccine candidate.

\section{Population coverage analysis}


The selected CTL and HTL epitopes covered $94.9 \%$ and $73.11 \%$ of the world population,

252 respectively. Importantly, CTL and HTL epitopes showed $98.63 \%$ population coverage worldwide

253 when considered in combination. The highest population coverage was found to be $99.99 \%$ in the

254 Latin American country, Peru (Fig. 7, Supplemental Data 2). In China, where the viral strain

255 (SARS-CoV-2) first appeared and had more devastating outbreaks, the population coverage for

256 CTL and HTL epitopes was $92.67 \%$ and $53.44 \%$, respectively with a combined coverage of $25796.59 \%$. SARS-CoV-2 is currently causing serious pandemics in different continents of the globe 258 including Italy, England, Spain, Iran, South Korea and United States of America where the 259 combined population coverage was found to be $98.8 \%, 99.44 \%, 95.35 \%, 98.48 \%, 99.19 \%$ and 260 99.35\%, respectively (Fig. 7a, Supplemental Data 2). In addition to geographical distribution, the 261 ethnic groups also found to be an important determinant for good coverage of the CTL and HTL 262 epitopes (Fig. 7b). Of the studied 147 ethnic groups, the Peru Amerindian had highest population 263 coverage for CTL (99.98\%) while the HTL epitopes had highest population coverage for Austria 264 Caucasoid (88.44\%) (Fig. 7b, Supplemental Data 2). Furthermore, 53.06\% of the ethnic groups 265 had a combined population coverage of more than $90.0 \%$ for both CTL and HTL epitopes.

\section{Expression prediction of the CoV-RMEN}

The length of the optimized codon sequence of the vaccine construct CoV-RMEN in $E$.

268 coli (strain K12) was 1,251 nucleotides. The optimized nucleotide sequence had a Codon 269 Adaptation Index (CAI) of 0.87 , and the average GC content of $50.26 \%$ showing the possibility of 270 good expression of the vaccine candidate in the E. coli host (Fig. 8a-c). Moreover, the evaluation 271 of minimum free energy for 25 structures of chimeric mRNA through Mfold'server showed that $272 \Delta \mathrm{G}$ of the best predicted structure for the optimized construct was $\Delta \mathrm{G}=-386.50 \mathrm{kcal} / \mathrm{mol}$. The 273 first nucleotides at $5^{\prime}$ did not have a long stable hairpin or pseudoknot. Therefore, the binding of 
274 ribosomes to the translation initiation site, and the following translation process can be readily

275 accomplished in the target host. These outcomes were in the agreement with data obtained from

276 the 'RNAfold'web server (Fig. 8 d,e) where the free energy was $-391.37 \mathrm{kcal} / \mathrm{mol}$.

277 After codon optimization and mRNA secondary structure analysis, the sequence of the 278 recombinant plasmid was designed by inserting the adapted codon sequences into pETite vector 279 (Lucigen, USA), which contains SUMO (Small Ubiquitin-like Modifier) tag and 6x-His tag 280 facilitating both the solubilization and affinity purification of the recombinant protein using 281 SnapGene software (Fig. 9). As alternative to E. coli for the expression system, HEK-293 282 eukaryotic cell line found promising for CoV-RMEN expression. The codon adaption index (CAI), $283 \mathrm{GC}$ content for this system were 1.0 and 61.60 respectively, which indicate high level of expression 284 of the vaccine construct in the HEK-293 cell line as well.

\section{Discussion}

SARS-CoV-2, the virus with high zoonotic importance and transmission rate, has spread 287 rapidly around the world and causes life-threatening COVID-19 (Gorbalenya et al., 2020). The number of SARS-CoV-2 infections, and subsequent deaths are increasing day by day (Tai et al., 2020), and thus, COVID-19 outbreak was declared as a public health emergency by the the world on its own way is trying to find out and/or develop an effective and safe vaccine against 292 this rapidly emerging pandemic SARS-CoV-2 (Abdelmageed et al., 2020). Although a good 293 number of vaccine candidates for COVID-19 are now under trials, some of them are advanced to 294 human trials (Lane, 2020), none has yet been declared to be effective and safe for prevention of 295 SARS-CoV-2 infections. Strikingly, no effective therapeutic drugs or vaccines are yet to be 
296 available for the treatment of SARS-CoV-2 patients (Hoque et al., 2020a). Through a

297 comprehensive genomic and proteomic study, we endeavor to desing an antigenic multi-epitope

298 (immunodominant) chimeric vaccine for SARS-CoV-2, named as CoV-RMEN (417 aa), which

299 will nullify the involvement of lab-escape viral transmission, reduce the cost, and may elicit

300 immunity by selectively stimulating antigen-specific B- and T-cells.

The novel approach of multi-epitope based (includes conserved multiple epitopes) vaccines

302 designing represents inducing specific cellular immunity, and highly potent neutralizing antibodies against infections (Dawood et al., 2019; Yong et al., 2019; Gralinski \& Menachery, 2020; Kibria et al., 2020). These epitope-based vaccines also provide increased safety and have the ability to focus on sustainable immune responses because of including conserved multiple epitopes. Unlike the full-length S protein, the RBD and NTD segments possess critical neutralizing domains without any non-neutralizing immunodominant region (ul Qamar et al., 2019; Gralinski \& Menachery, 2020; Shang et al., 2020; Wrapp et al., 2020). Mutations on the RBD may enable the new strains to escape neutralization by established RBD-targeting antibodies, hence other functional regions, especially the NTD, should be considered for developing an effective vaccine 311 as well (Wang et al., 2019; Zhou et al., 2019). Besides, combined administration of RBD and NTD 312 proteins induced highly potent neutralizing antibodies and long-term protective immunity in 313 animal models (Song et al., 2018). Considering the safety and effectiveness perspectives, the RBD 314 and NTD are more promising candidates in the development of SARS-CoV-2 vaccines over the 315 full-length $\mathrm{S}$ protein. The presence of $\mathrm{E}$ and $\mathrm{M}$ proteins on the envelope can augment the immune 316 response against SARS-CoV (Millet \& Whittaker, 2015; Almofti et al., 2018) and thus, considered

317 for suitable candidate for vaccine development (Yong et al., 2020; Ahmed et al., 2020; Gralinski 318 \& Menachery, 2020). Thus, antibodies against the immunologically substantial epitopes of S, M 
319 and E proteins of SARS-CoV-2 would provide protective immunity to the infection (Yong et al.,

320 2020; Ahmed et al., 2020; Gralinski \& Menachery, 2020; Shang et al., 2020). Therefore, the

321 immune response targeting the RBD and/or NTD of the S glycoprotein, $\mathrm{M}$ and E proteins of SARS-

$322 \mathrm{CoV}-2$ would be an important prophylactic and therapeutic interventions, which can be tested

323 further in suitable models before clinical trials (Chan et al., 2020).

Effective immunity to viral infections is significantly dependent on activation of both B-

325 and T-cells (Shi et al., 2015; Shey et al., 2019). Therefore, inducing specific humoral or cellular immunity against pathogens, an ideal vaccine should contain both B-cell and T-cell epitopes. Our analyses revealed that selected RBD and NTD regions of the CoV-RMEN contain ample amount of high-affinity B-cell, MHC Class I, MHC Class II and interferon- $\gamma($ IFN- $\gamma$ ) epitopes with high antigenicity scores. Moreover, membrane B-cell epitope (MBE) and envelope B-cell epitope (EBE) enhanced the overall stability, immunogenicity and antigenicity of the CoV-RMEN. The

331 development of memory B-cells and T-cells was evident, with memory in B-cells lasting for 332 several months. These finding opposed to several earlier reports where T-cell mediated immune response was considered a long-lasting response compared to B-cells (Abdelmageed et al., 2020;

334 Wrapp et al., 2020). Another engrossing finding of this study was the development of Th1 response 335 which enhances the growth and proliferation of B- cells augmenting the adaptive immunity 336 (Carvalho et al., 2002). If a strong B-cell response occurred in animal trials (mice or rabbit), these 337 antibodies could be used in diagnostic purposes, as they should recognize the prominent antigens 338 on the viral surface (Kibria et al., 2020). Moreover, CD8+ and CD4+ T-cell responses play major 339 role in antiviral immunity (Abdelmageed et al., 2020). Another crucial fact is that Toll-Like 340 Receptors (TLRs) can effectively bind with spike protein of the CoV (Totura et al., 2015; Zander 
341 et al., 2017), and might play an important role in the innate immune response to SARS-CoV-2

342 infection (Shahabi et al., 2020).

343 The physicochemical properties also revealed the chimera as a basic or alkaline protein

$344(\mathrm{pI}=8.71)$ and would be thermostable upon expression, and thus, our proposed vaccine CoV-

345 RMEN would be best suited for worldwide use in different endemic areas (Shey et al., 2019; ul

346 Qamar et al., 2019). The structural forms (secondary and tertiary) of the CoV-RMEN, when tested

347 as the synthetic peptides, showed the ability to fold into their native structure, hence could mimic

348 the natural infection by SARS-CoV-2 (Almofti et al., 2018). The refined tertiary (3D) structure of

349 the final vaccine construct markedly presented the desirable structural features based on

350 Ramachandran plot predictions (Shey et al., 2019; Srivastava et al., 2019). Molecular docking

351 analysis showed that predicted chimeric protein can establish stable protein-protein interactions

352 with TLRs (TLR-2, TLR-3, TLR-4) (Totura et al., 2015). An efficient activation of surface

353 molecules of the CoV-RMEN is very crucial for immune activation of dendritic cells, and

354 subsequent antigen processing and presentation to CD4+ and CD8+ T-cells via MHC-II and MHC-

355 1, respectively (Shi et al., 2015; Shey et al., 2019; Shang et al., 2020). The molecular dynamics

356 simulation also revealed that the docked CoV-RMEN-TLRs complexes were stable, and had more

357 binding affinity TLR-3 and TLR-4 (Abraham et al., 2015). Furthermore, the CoV-RMEN showed

358 good antigenicity scores on Vaxijen v2.0 and ANTIGENpro indicating that these peptide

359 sequences are supposed to be highly antigenic in nature (Shey et al., 2019). The non-allergenic

360 properties of the CoV-RMEN further strengthens its potential as a vaccine candidate (Shey et al.,

361 2019; ul Qamar et al., 2019).

Immune simulation of the CoV-RMEN exhibited expected results consistent with typical

363 immune responses, and there was a growing immune responses after the recurrent antigen 
364 exposures (Fig. 6). The antiviral cytokine IFN- $\gamma$ and cell stimulatory IL-2 level significantly

365 increased, which also contribute to the subsequent immune response after vaccination in host 366 (Almofti et al., 2018). This indicates high levels of helper T-cells and consequently efficient 367 antibody production, supporting a humoral response (Shey et al., 2019). A lower $\mathrm{IC}_{50}$ value 368 indicates higher binding affinity of the epitopes with the MHC class I and II molecules. While 369 most of the previous studies (Sakib et al., 2014; Adhikari et al., 2018) reported that a binding 370 affinity $\left(\mathrm{IC}_{50}\right)$ threshold of $250 \mathrm{nM}$ identifies peptide binders recognized by T-cells, and this 371 threshold can be used to select peptides, we kept binding affinity within $50 \mathrm{nM}$ to get better 372 confidence level in predicting epitopes for MHC-I and MHC-II alleles.The Simpson index 373 estimated clonal specificity suggested a possible diverse immune response and this is plausible 374 considering the generated chimeric peptide is composed of sufficient B- and T-cell epitopes (Fig. 375 6). The interaction between T-cell epitopes, and their respective HLA alleles revealed 377 significant binding affinity reflecting the immune activation of B- and T-cells as supported by 378 other reports (Srivastava et al., 2019; Jaimes et al., 2020). T-cell epitopes from RBD and NTD 379 regions showing high interaction with HLA alleles covered more than $98 \%$ of the world population 380 with different ethnic groups, and these findings corroborated with many of earlier studies (Huang 381 et al., 2007; Jaimes et al., 2020; ul Qamar et al., 2019). The incorporation of GG and EGGE linkers 382 between the predicted epitopes of the CoV-RMEN produced sequences with minimized junctional 383 immunogenicity, and allowed the rational design construction of a potent multi-epitope vaccine 384 (Huang et al., 2007; Badawi et al., 2016; Shey et al., 2019; Srivastava et al., 2019). The 385 glycosylation of the surface antigens helps the enveloped viruses evade recognition by the host 386 immune system, and can influence the ability of the host to raise an effective adaptive immune 
387 response (Pereira et al., 2018) or even be exploited by the virus to enhance infectivity (Wolfert et

388 al., 2013). Moreover, some antibodies such as mAb 8ANC195 have evolved to recognize peptide

389 epitope with no dependence on glycan binding (Kong et al., 2015). However, there is no data

390 available for antibodies specific to spike glycoproteins of SARS-CoV-2, whether their recognition

391 is interfered by the glycosylation of spike or may either be strengthened by sugars close to the

392 peptide epitope, or not interfered by sugar modification (Zhou et al., 2020b). Most of the epitopes

393 of the CoV-RMEN harbor no glycoside except NTD region (Watanabe et al, 2020). Most of the

394 glycans of the NTD epitopes present at the terminii of the putative HLA antigens, and may not

395 interfere with antigen presentation in an HLA complex (Grant et al., 2020). Integrity of the ACE2

396 receptor of the RBD, envelope protein B-cell epitope (EBE) and membrane protein B-cell epitope

397 (MBE) in the CoV-RMEN suggests that the vaccine may maintain efficacy despite antigenic drift 398 and glycosylation phenomena, as long as the virus continues to target the same host receptor (Grant 399 et al., 2020).

One of the first steps in validating a candidate vaccine is to screen for immunoreactivity

401 through serological analysis. This requires the expression of the recombinant protein in a suitable

402 host. As we focused on the epitopes without the glycosylation or non-significant glycosylation,

403 high-level expression of the vaccine was optimized into well-established and cost-effective

404 prokaryotic expression system E. coli $\mathrm{K}-12$ strain as the first choice using the plasmid pETite 405 containing SUMO (Small Ubiquitin-like Modifier) tag and 6x-His tag facilitated both the 406 solubilization and affinity purification of the recombinant protein (Biswal et al., 2015). Codon 407 optimization of the CoV-RMEN revealed its high-level expression in E. coli (strain K12). Stable 408 mRNA structure, codon adaptability index $(0.87)$, and the GC content $(50.26 \%)$ were favourable 409 for high-level expression of the protein in the bacterium. After successful cloning of the gene, the 
410 recombinant plasmid can be propagated efficiently using E. coli cells, and subsequent protein

411 expression can be performed in E. coli K-12 strain using IPTG (Isopropyl $\beta$-d-1-

412 thiogalactopyranoside) induction, and cultivation at $28^{\circ} \mathrm{C}$ as also reported earlier (Biswal et al., 413 2015).

As alternative to E. coli, eukaryotic cell line, HEK-293 was considered for CoV-RMEN

415 expression. The codon adaption index (CAI), GC content were 1.0 and 61.60 respectively, wihch 416 indicate high level of expression of vaccine construct in the HEK-293 cell line. In this case, pSec 417 Tag2 Mammalian expression vector could be used, which have secretion signal from the V-J2-C 418 region of the mouse Ig kappa-chain for efficient secretion of the recombinant protein, C-terminal 419 poly-histidine (6xHis) tag for rapid purification with C-termnal c-myc epitope for detection with 420 an Anti-myc antibody. To remove the affinity and detection tags (His tag and c-myc epitope) after 421 purification, Factor Xa clevage site (LVPR $\downarrow$ GS ) could be added to the C-terminal of the CoV422 RMEN (Waugh et al., 2011).

\section{Conclusions}

This multi-epitope peptide vaccine candidate, CoV-RMEN possesses potential epitopes

425 form the RBD and NTD segments of spike (S), M and E proteins retaining potential antigenicity 426 and non-allergenicity properties. This chimera, suitable for high-level expression and cloning, 427 includes potential CTL, HTL and B-cell epitopes ensuring humoral and cell mediated immunity, 428 as well as predicted immune-simulation refers to increased production of immunoglobulins and 429 cytokines. Molecular docking and dynamic simulation of the CoV-RMEN with the immune 430 receptors (TLRs) predicted strong binding affinity, in particular with TLR3 and TLR4. 431 Remarkably, the CoV-RMEN had more than $90.0 \%$ world population coverage for different ethnic 
432 groups. The limitations posed by fewer number of SARS-CoV-2 geneome sequence data which

433 tends to mutate frequently may not affect our analysis since we included four peptides from three

434 major proteins of SARS-CoV-2 genome in a multi-epitope vaccine with the high conservancy.

435 However, future in vitro and in vivo studies are required to assess the potentiality of the designed

436 vaccine candidate to induce a positive immune response against SARS-CoV-2 infections, and also

437 to validate the results obtained herein through immuno-informatics analyses.

438 Methods

439 Sequence retrieval and structural analysis

440 A total of 250 partial and complete genome sequences of SARS-CoV-2 were retrieved 441 from NCBI (National Center for Biotechnology Information,

442 https://www.ncbi.nlm.nih.gov/protein) (Table S5). We aligned these sequences through MAFFT

443 online server (https://mafft.cbrc.jp/alignment/server/) using default parameters, and Wu-Kabat 444 protein variability was analyzed (Fig. S9) in protein variability server 445 (http://imed.med.ucm.es/PVS/) for SARS-CoV-2 NCBI reference genome (Accession no : 446 NC_045512.2). We retrieved the S protein sequences of the SARS-CoV and MERS-CoV from the 447 whole genome reference sequences of the respective three viruses from the NCBI database. 448 Moreover, the S proteins of SARS-CoV (GenBank accession no: NC_004718.3), MERS-CoV 449 (GenBank accession no: NC_019843.3) and SARS-CoV-2 were structurally compared using 450 SWISS homology modeling (Waterhouse et al., 2018) based on the protein databank (PDB) 451 templates 6acd, 5w9h and 6vsb, respectively aligned using PyMOL (Faure et al., 2019), and 452 observed for heterogeneous domains in the conformations. The models were optimized by energy 453 minimization using the GROMOS96 program (Van Gunsteren et al., 1996) implemented within 
454 the Swiss-PdbViewer, version 4.1.0 (Guex \& Peitsch, 1997). The Ramachandran plots of the

455 derived models were evaluated using a PROCHECK (version 3.5)-server to check the

456 stereochemical properties of the modeled structures (Laskowski et al., 1993). All further analyses

457 including epitopes selection, antigenicity and allergenicity profiles, molecular docking and final

458 vaccine construct were performed based on the NCBI reference genome of SARS-CoV-2.

\section{Screening for $\mathbf{B}$ and $\mathbf{T}$ cell epitopes}

460 Conformational B-cell epitopes on the $\mathrm{S}$ protein were predicted by ElliPro

461 (http://tools.iedb.org/ellipro/) with the minimum score value set at 0.4 while the maximum distance

462 selected as $6 \AA$ (Kringelum et al., 2012). The linear B-cell epitopes of RBD and NTD regions of

463 S protein, full length E and M proteins were predicted by "BepiPred Linear Epitope Prediction"

464 (Larsen et al., 2006), and ABCPred with default parameters (Saha \& Raghava, 2006). To find out

465 the most probable peptide-based epitopes with better confidence level, antigenecity of the

466 predicted peptides were further verified using VaxiJen antigenicity scores (Kringelum et al., 2012).

467 The Kolaskar and Tongaonkar antigenicity profiling from IEDB analysis resource was also used

468 for the RBD and NTD segments, E and M proteins (Kolaskar \& Tongaonkar, 1990).

469 CTL epitopes, proteasomal cleavage and transporter associated with antigen processing

470 (TAP), and HTL epitopes of the SARS-CoV-2 S, E and M proteins were predicted using IEDB

471 resource tool Proteasomal cleavage/TAP transport/MHC class I combined predictor

472 (http://tools.iedb.org/main/tcell/) with all default parameters . Moreover, the HTL epitopes of the

473 proteins were screened using the IEDB tool "MHC-II Binding Predictions"

474 (http://tools.iedb.org/mhcii/). Threshold for strong binding peptides $\left(\mathrm{IC}_{50}\right)$ was set at $50 \mathrm{nM}$ to

475 determine the binding and interaction potentials of helper T-cell epitopes and both major 
476 histocompatibility complex (MHC) class I and alleles (Shi et al., 2015). Top five HLA epitopes

477 for each RBD and NTD segments were docked against the respective HLA (MHC- I and MHC-

478 II ) allele binders by interaction similarity-based protein-peptide docking system GalaxyPepDock

479 of the GalaxyWeb, docked HLA-epitope complexes were refined in GalaxyRefineComplex and

480 binding affinity $(\Delta \mathrm{G})$ was determined PROtein binDIng enerGY prediction (PRODIGY) tool (Xue

481 et al., 2016).

$482 \quad$ IFN- $\gamma$-inducing epitope prediction

483 Potential IFN- $\gamma$ epitopes of all the selected antigenic sites of RBD, NTD, envelope protein 484 B-cell epitope (EBE), and membrane protein B-cell epitope (MBE) were predicted by 485 "IFNepitope" server (http://crdd.osdd.net/raghava/ ifnepitope/scan.php). To identify the set of 486 epitopes associated with MHC alleles that would maximize the population coverage, we adopted 487 the "Motif and SVM hybrid" (MERCI: Motif-EmeRging and with Classes-Identification, and 488 SVM) approach. The prediction is based on a dataset of IFN- $\gamma$-inducing and IFN- $\gamma$-noninducing 489 MHC allele binders (Dhanda et al., 2013).

Design and construction of multi-epitope vaccine candidate (CoV-RMEN)

492 previously published peptide vaccine development protocol for different emerging infectious 493 diseases like SARS and MERS (Shi et al., 2015; Badawi et al., 2016; Almofti et al., 2018; Shey et 494 al., 2019; Srivastava et al., 2019. Ul Qamar et al., 2019). The multi-epitope protein was constructed 495 by positioning the selected RBD, NTD, MBE and EBE aa sequences linked with short, rigid and 496 flexible linkers GG. To develop highly immunogenic recombinant proteins, two universal T-cell 
497 epitopes were used, namely, a pan-human leukocyte antigen DR-binding peptide (PADRE)

498 (Agadjanyan et al., 2005), and an invasin immunostimulatory sequence taken from Yersinia

499 (Invasin) (Li et al., 2015) were used to the $\mathrm{N}$ and $\mathrm{C}$ terminal of the vaccine construct respectively,

500 linked by EGGE.

501 Secondary and tertiary structure of the CoV-RMEN

502

Chou and Fasman secondary structure prediction server (CFSSP:

503 https://www.biogem.org/tool/chou-fasman/), and RaptorX Property

504 (http://raptorx.uchicago.edu/StructurePropertyPred/predict/) web-servers were used for secondary 505 structure predictions (Källberg et al., 2014). The tertiary structure of the CoV-RMEN was built in 506 homology/analogy recognition

engine

(Phyre2)

507 (http://www.sbg.bio.ic.ac.uk/ phyre2/html/page.cgi?id=index) web-server. The 3D model was

508 refined in a three-step process, initially energy minimization using the GROMOS96 program

509 implemented within the Swiss-PdbViewer (version 4.1.0). After energy minimization, the model

510 was refined using ModRefiner (https://zhanglab.ccmb.med.umich.edu/ModRefiner/) and then

511 GalaxyRefine server (http://galaxy.seoklab.org/cgi-bin/submit.cgi?type=REFINE).

512 Furthermore, the local structural quality of the CoV-RMEN was refined with GalaxyRefine

513 server, and ProSA-web (https://prosa.services.came.sbg.ac.at/prosa.php) was used to calculate 514 overall quality score for the refined structure. The ERRAT server 515 (http://services.mbi.ucla.edu/ERRAT/) was also used to analyze non-bonded atom-atom 516 interactions compared to reliable high-resolution crystallography structures. A Ramachandran plot 517 was obtained through the RAMPAGE server (Lovell et al., 2003).

\section{Physiochemical properties prediction of CoV-RMEN}


520 various physiochemical parameters of the CoV-RMEN including aa residue composition, 521 molecular weight, theoretical pI, instability index, in vitro and in vivo half-life, aliphatic index, 522 and grand average of hydropathicity (GRAVY). The solubility of the multi-epitope vaccine peptide 523 was evaluated using the Protein-Sol server (https://protein-sol.manchester.ac.uk/).

\section{Antigenicity, allergenicity and Immune simulation of the CoV-RMEN}

525

526

527

528

529

530

531

532

533

534

535

536

537 538

539

540

VaxiJen 2.0 and ANTIGENpro (http://scratch.proteomics.ics.uci.edu/) web-servers were used to predict the antigenicity of the CoV-RMEN while the AllerTOP 2.0 (http://www.ddgpharmfac.net/AllerTOP) and AllergenFP (http://ddg-pharmfac.net/AllergenFP/) web-servers were used to predict vaccine allergenicity. Further, the immunogenicity and immune response profile of the CoV-RMEN were characterized by in silico immune simulations using the C-ImmSim server (http://150.146.2.1/C-IMMSIM/index.php) under default parameters with time steps set at 1, 84, and 170 (each time step is 8 hours and time step 1 is injection at time $=0$ ). Therefore, three shots were given at four weeks apart.

\section{Molecular docking and dynamics of the CoV-RMEN with TLRs}

Molecular docking of the CoV-RMEN with the TLR2 (PDB ID:3D3I), TLR3 (PDB ID: 1ZIW) and TLR4 (PDB ID: 4G8A) receptors was performed using the High Ambiguity Driven DOCKing (HADDOCK, version 2.4) (De Vries et al., 2010) web-server to evaluate the interaction between ligand (CoV-RMEN) and receptors (TLRs) leading to an enhanced immune response. CPORT (https://milou.science.uu.nl/services/CPORT/) was used to predict active interface residues between the CoV-RMEN and TLRs. For the analysis of the stable complex formation, all the complexes (TLRs with CoV-RMEN) were subjected to molecular dynamics (MD) simulation 
541 by Gromacs 2020.2 using OPLS-AA (Abraham et al., 2015; Jorgensen et al., 1996). Finally, the

542 binding affinities of the docked chimeric protein-TLRs complexes were predicted using the

543 PRODIGY (PROtein binDIng enerGY prediction) (https://nestor.science.uu.nl/prodigy/) web-

544 server.

545 Analysis of cDNA and mRNA for cloning and expression of CoV-RMEN

546 Reverse translation and codon optimization were performed using the GenScript Rare

547 Codon Analysis Tool (https://www.genscript.com/tools/rare-codon-analysis) in order to express

548 the CoV-RMEN in the E. coli (strain K12). Stability of the mRNA was verified using two different

549 tools, namely RNAfold (http://rna.tbi.univie.ac.at/cgi-bin/RNAWebSuite/RNAfold.cgi) and the

550 mfold (http://unafold.rna.albany.edu/?q=mfold) web-servers. The optimized gene sequence

551 of CoV-RMEN will be artificially synthesized having N-terminal recombinant human rhinovirus

552 (HRV 3C) protease site (LEVLFQ $\downarrow$ GP) and cloned the final construct into pETite vector (Lucigen,

553 USA) through enzyme-free method (Waugh et al., 2011). Finally, the sequence of the recombinant

554 plasmid was designed by inserting the adapted codon sequences into pETite vector using

555 SnapGene software (from Insightful Science; available at snapgene.com). As an alternative to $E$.

556 coli, eukaryotic expression system HEK-293 was optimized using similar analysis for the vaccine 557 production.

\section{Population coverage by CTL and HTL epitopes}

The predicted T-cell epitopes were shortlisted based on the aligned Artificial Neural

560 Network (ANN) with half-maximal inhibitory concentration $\left(\operatorname{annIC}_{50}\right)$ values up to $50 \mathrm{nM}$. The

561 IEDB "Population Coverage" tool (http://tools.iedb.org/population/) was used to determine the 562 world human population coverage by the shortlisted CTL and HTL epitopes (Bui et al., 2006). We 
563 used OmicsCircos to visualize the association between world population and different ethnic 564 groups (Hoque et al., 2020b).

565

566

567

568

569

570

571

572

573

574

575

576

577

578

579 


\section{Competing Interest}

581 The authors declare no competing interests.

\section{Author contributions}

583

MSR, MNH, and MRI done the overall study and also draft the manuscript. SA drafted 584 some parts of results and discussion. MNH finally compiled the manuscript. OS, and MAS helped 585 in final discussion and reference indexing. ASMRUA, MMR, MS and MAH contributed 586 intellectually to the interpretation and presentation of the results. MS and MAH developed the 587 concept of peptide vaccine development. Finally, all authors have approved the manuscript for 588 submission.

\section{Acknowledgements}

590 The authors thank Joynob Akter Puspo, Masuda Akter and Israt Islam (MS student), and 591 DR. Kazi Alamgir Hossain (PhD Fellow) of the Microbial Genetics and Bioinformatics 592 Laboratory, Department of Microbiology, University of Dhaka for their cooperation, suggestions 593 and overall encouragement during the preparation of the manuscript. The authors also extend their 594 thanks to those who made their sequence data available in NCBI.

\section{Supplemental Information}

596 Supplemental information supporting the findings of the manuscript could be found from

597 the publishers (PeerJ), and from the corresponding author on request.

\section{Data Availability}

599 This in silico study utilizes genomic sequence data available in NCBI database (Accession no :

600 NC_045512.2, NC_004718.3, and NC_019843.3). 


\section{References}

604 Abdelmageed MI, Abdelmoneim AH, Mustafa MI, Elfadol NM, Murshed NS, Shantier SW, 605

606

607

608

609

610

611

612

613

614

615

616

617

618

619

620

621

622

623

624

625 Makhawi AM. . 2020. Design of a Multiepitope-Based Peptide Vaccine against the E Protein of Human COVID-19: An Immunoinformatics Approach. BioMed Research International 2683286, https://doi.org/10.1155/2020/2683286.

Abraham MJ, Murtola T, Schulz R, Páll S, Smith JC, Hess B, Lindahl E. 2015. GROMACS: High performance molecular simulations through multi-level parallelism from laptops to supercomputers. SoftwareX 1-2(2), 19-25.

Adhikari UK, Tayebi M, Rahman MM. 2018. Immunoinformatics approach for epitope-based peptide vaccine design and active site prediction against polyprotein of emerging oropouche virus. Journal of Immunology Research 6718083, https://doi.org/10.1155/2018/6718083.

Agadjanyan MG, Ghochikyan A, Petrushina I, Vasilevko V, Movsesyan N, Mkrtichyan M, Tommy S, Cribbs DH. 2005. "Prototype Alzheimer's disease vaccine using the immunodominant B cell epitope from $\beta$-amyloid and promiscuous T cell epitope pan HLA DR-binding peptide." The Journal of Immunology 174(3), 1580-1586.

Ahmed SF, Quadeer AA, McKay MR. 2020. Preliminary identification of potential vaccine targets for the COVID-19 coronavirus (SARS-CoV-2) based on SARS-CoV immunological studies. Viruses 12(3), 254.

Akhand MRN, Azim KF, Hoque SF, Moli MA, Joy BD, Akter H, Ibrahim KA, Nadim A, Hasan M. 2020. Genome based Evolutionary study of SARS-CoV-2 towards the Prediction of Epitope Based Chimeric Vaccine. bioRxiv doi: https://doi.org/10.1101/2020.04.15.036285. 
626 Almofti YA, Abd-elrahman KA, Gassmallah SAE, Salih MA. 2018. Multi Epitopes Vaccine

627 Prediction against Severe Acute Respiratory Syndrome (SARS) Coronavirus Using

628 Immunoinformatics Approaches. American Journal of Microbiological Research 6(3),

629 94-114.

630 Badawi MM, SalahEldin MA, Suliman MM, AbduRahim SA, elghafoor Mohammed AA,

631 SidAhmed ASA, Marwa MO, Salih MA. 2016. In Silico Prediction of a Novel

632 Universal Multi-epitope Peptide Vaccine in the Whole Spike Glycoprotein of MERS

633 CoV. American Journal of Microbiological Research 4(4), 101-21.

634 Biswal JK, Bisht P, Mohapatra JK, Ranjan R, Sanyal A, Pattnaik B. 2015. "Application of a

635 recombinant capsid polyprotein (P1) expressed in a prokaryotic system to detect

636 antibodies against foot-and-mouth disease virus serotype O." Journal of Virological

637 Methods 215, 45-51.

638 Bui HH, Sidney J, Dinh K, Southwood S, Newman MJ, Sette A. 2006. Predicting population

639 coverage of T-cell epitope-based diagnostics and vaccines. BMC Bioinformatics

$640 \quad \mathbf{7 ( 1 )}, 153$.

641 Carvalho LH, Sano GI, Hafalla JC, Morrot A, De Lafaille MAC, Zavala F. 2002. IL-4-

642 secreting CD4+ T cells are crucial to the development of CD8+ T-cell responses

643 against malaria liver stages. Nature Medicine 8, 166-70.

644 Chakraborty S, Chakravorty R, Ahmed M, Rahman A, Waise TM, Hassan F, Mustafizur R,

645 Shamsuzzaman S. 2010. A computational approach for identification of epitopes in

646 dengue virus envelope protein: a step towards designing a universal dengue vaccine

647 targeting endemic regions. In silico Biology 10(5-6), 235-246. 
648 Chan JFW, Kok KH, Zhu Z, Chu H, To KKW, Yuan S, Yuen KY. 2020. Genomic

649 characterization of the 2019 novel human-pathogenic coronavirus isolated from a 650 patient with atypical pneumonia after visiting Wuhan. Emerging Microbes and $651 \quad$ Infections 9, 221-236.

652 Dawood RM, Moustafa RI, Abdelhafez TH, El-Shenawy R, El-Abd Y, El Din NGB, Jean D, 653 El Awady MK. 2019. A multiepitope peptide vaccine against HCV stimulates 654 neutralizing humoral and persistent cellular responses in mice. BMC Infectious

655 Diseases 19, 932.

656

De Vries SJ, Van Dijk M, Bonvin AM. 2010. The HADDOCK web server for data-driven biomolecular docking. Nature Protocol 5(5), 883.

Dhanda SK, Vir P, Raghava GP. 2013. "Designing of interferon-gamma inducing MHC class659 II binders." Biology Direct 8(1), 30.

660

661 tumour antigens and subunit vaccines. BMC Bioinformatics 8(1), 4. use in peptide based vaccines. Current Pharmaceutical Design 16(28), 3149-3157. AG. 2019. iPBAvizu: a PyMOL plugin for an efficient 3D protein structure 666 superimposition approach. Source Code for Biology and Medicine 14, 5.

667 Gasteiger E, Hoogland C, Gattiker A, Wilkins MR, Appel RD, Bairoch A. 2005. Protein 668 identification and analysis tools on the ExPASy server. The Proteomics Protocols 669 Handbook, Springer: 571-607. 
670 Gorbalenya AE, Baker SC, Baric RS, de Groot RJ, Drosten C, Gulyaeva AA, Haagmans BL, 671 Lauber C, Leontovich AM, Neuman BW, Penzar D. 2020. The species Severe acute 672 respiratory syndrome-related coronavirus: classifying 2019-nCoV and naming it 673 SARS-CoV-2. Nature Microbiology 5(4), 536-544, doi:10.1038/s41564-020-0695-z.

674 Gralinski LE, Menachery VD. 2020. Return of the coronavirus: 2019-nCoV. Viruses 12, 135.

675 Grant OC, Montgomery D, Ito K, Woods RJ. 2020. Analysis of the SARS-CoV-2 spike 676 protein glycan shield: implications for immune recognition. bioRxiv doi: https://doi.org/10.1101/2020.04.07.030445.

Guex N, Peitsch MC. 1997. "SWISS-MODEL and the Swiss-Pdb Viewer: an environment for

679 comparative protein modeling." Electrophoresis 18(15), 2714-2723.

680

Hasan A, Hossain M, Alam J. 2013. A computational assay to design an epitope-based Peptide vaccine against Saint Louis encephalitis virus. Bioinformatics and Biology Insights 7,

682 BBI-S13402.

Hoque MN, Istiaq A, Clement RA, Sultana M, Crandall KA, Siddiki AZ, Hossain M A. 2019. Metagenomic deep sequencing reveals association of microbiome signature with 685 functional biases in bovine mastitis. Scientific Reports 9, 13536.

686 Hoque MN, Chaudhury A, Akanda MAM, Hossain MA, Islam MT. 2020a. Genomic Diversity 687 and Evolution, Diagnosis, Prevention, and Therapeutics of the Pandemic COVID-19 Disease. Preprints 2020040359, DOI: 10.20944/preprints202004.0359.v1. Siddiki AMAM, Crandall KA, Hossain MA. 2020b. Insights into the Resistome of Bovine Clinical Mastitis Microbiome, a Key Factor in Disease Complication. Frontiers in Microbiology 11, 860, doi: 10.3389/fmicb.2020.00860. 
693 Huang H, Hao S, Li F, Ye Z, Yang J, Xiang J. 2007. CD4(+) Th1 cells promote CD8(+) Tc1

694 cell survival, memory response, tumor localization and therapy by targeted delivery of

695 interleukin 2 via acquired pMHC I complexes. Immunology 120, 148-159.

696 HuiDS IAE, Madani TA, Ntoumi F, Koch R, Dar O. 2020. The continuing 2019-nCoV

697 epidemic threat of novel coronaviruses to global health-The latest 2019 novel

698 coronavirus outbreak in Wuhan, China. International Journal of Infectious Diseases

$699 \quad 91,264-266$.

700 Jaimes JA, André NM, Millet JK, Whittaker GR. 2020. Structural modeling of 2019-novel 701 coronavirus $(\mathrm{nCoV})$ spike protein reveals a proteolytically-sensitive activation loop as

702 a distinguishing feature compared to SARS-CoV and related SARS-like 703 coronaviruses. arXiv Preprint, arXiv:2002.06196.

704 Källberg M, Margaryan G, Wang S, Ma J, Xu J. 2014. RaptorX server: a resource for 705 template-based protein structure modeling. In Protein Structure Prediction (pp. 17706 27), Humana Press, New York, NY.2014.

707 Kibria, KM K, Ullah H, Miah M. 2020. The multi-epitope vaccine prediction to combat 708 Pandemic SARS-CoV-2, an immunoinformatic approach. Preprints, doi: 709 10.21203/rs.3.rs-21853/v1.

710 Kolaskar A, Tongaonkar PC. 1990. "A semi-empirical method for prediction of antigenic 711 determinants on protein antigens." FEBS Letters 276(1-2), 172-174.

712 Kong L, Torrents de la Peña A, Deller MC, Garces F, Sliepen K, Hua Y, Stanfield RL, Sanders 713 RW, Wilson IA. 2015. Complete epitopes for vaccine design derived from a crystal structure of the broadly neutralizing antibodies PGT128 and 8ANC195 in complex 

10), 2099-108.

717 Kringelum JV, Lundegaard C, Lund O, Nielsen M. 2012. Reliable B cell epitope predictions: 718 Impacts of method development and improved benchmarking. PLOS Computational Biology 8(12), e1002829.

720 Lane R. 2020. Sarah Gilbert: carving a path towards a COVID-19 vaccine. Lancet (London, 721 England) 395(10232), 1247.

722 Larsen JEP, Lund O, Nielsen M. 2006. Improved method for predicting linear B-cell 723 epitopes. Immunome Research 2(1), 2.

724 Laskowski RA, MacArthur MW, Moss DS, Thornton JM. 1993 "PROCHECK: a program to 725 check the stereochemical quality of protein structures." Journal of Applied Crystallography 26(2), 283-291.

727 Le TT, Andreadakis Z, Kumar A, Roman RG, Tollefsen S, Saville M, Mayhew S. 2020. The COVID-19 vaccine development landscape. Nature Reviews Drug Discovery 19(5), $305-6$.

731

732 domain of Yersinia enterocolitica invasin enhances the efficacy of classical swinefever-vectored vaccine based on human adenovirus. Journal of Biosciences 40, 7990. Lovell SC, Davis IW, Arendall III WB, De Bakker PI, Word JM, Prisant MG, Richardson, JS, Richardson DC. 2003. "Structure validation by C $\alpha$ geometry: $\phi, \psi$ and C $\beta$ deviation." Proteins: Structure, Function, Bioinformatics 50(3), 437-450. 
737 Lu R, Zhao X, Li J, Niu P, Yang B, Wu H, Wang W, Song H, Huang B, Zhu N, Bi Y. 2020.

738 Genomic characterisation and epidemiology of 2019 novel coronavirus: implications

739 for virus origins and receptor binding. The Lancet 395(10224), 565-574.

740 Millet JK, Whittaker GR. 2015. "Host cell proteases: Critical determinants of coronavirus

741 tropism and pathogenesis." Virus Research 202, 120-134.

742 Pallesen J, Wang N, Corbett KS, Wrapp D, Kirchdoerfer RN, Turner HL, Cottrell CA, Becker 743 MM, Wang L, Shi W, Kong WP. 2017. Immunogenicity and structures of a rationally

744

745 designed prefusion MERS-CoV spike antigen. Proceedings of the National Academy of Sciences USA,114(35), E7348-E7357.

746

747

748

749 of Clinical Medicine 9(3), 623.

750

Pereira MS, Alves I, Vicente M, Campar A, Silva MC, Padrão NA, Pinto V, Fernandes Â,

751

752 Dias AM, Pinho SS. 2018. Glycans as Key Checkpoints of T Cell Activity and Function. Frontiers in Immunology 9, 2754, doi: 10.3389/fimmu.2018.02754. Pang J, Wang MX, Ang IYH, Tan SHX, Lewis RF, Chen JIP, Gutierrez RA, Gwee SXW, Chua PEY, Yang Q, Ng XY. 2020. Potential rapid diagnostics, vaccine and therapeutics for 2019 novel Coronavirus (2019-ncoV): a systematic review. Journal 755 Sakib MS, Islam M, Hasan AKM, Nabi AHM. 2014. Prediction of epitope-based peptides for 756 the utility of vaccine development from fusion and glycoprotein of nipah virus using in silico approach. Advances in Bioinformatics

402492, https://doi.org/10.1155/2014/402492. 
Schoeman D, Fielding BC. 2019. Coronavirus envelope protein: current knowledge. Virology Journal 16, 69. N, Masoompour SM. 2020. Therapeutic approaches for COVID-19 based on the

Shang W, Yang Y, Rao Y, Rao X. 2020. The outbreak of SARS-CoV-2 pneumonia calls for viral vaccines. npj Vaccines $\mathbf{5}, 18$.

Sheridan C. 2020. Questions remain following first COVID-19 vaccine results. Nature Biotechnology doi: https://www.nature.com/articles/d41587-020-00015-x. Ngale FN, Vanhamme L, Souopgui J. 2019. In-silico design of a multi-epitope vaccine candidate against onchocerciasis and related filarial diseases. Scientific Reports 9(1), $1-18$. Approach Applied to Emerging Infectious Diseases. PLoS One 10(12), e0144475. expression of membrane protein augments the specific responses induced by SARSCoV nucleocapsid DNA immunization. Molecular Immunology 43, 1791-1798. e1007236. 
782 Srivastava S, Kamthania M, Kumar Pandey R, Kumar Saxena A, Saxena V, Kumar Singh S, 783 Kumar Sharma R, Sharma N. 2019. Design of novel multi-epitope vaccines against severe acute respiratory syndrome validated through multistage molecular interaction and dynamics. Journal of Biomolecular Structure and Dynamics 37(16), 4345-4360. the receptor-binding domain (RBD) of 2019 novel coronavirus: implication for development of RBD protein as a viral attachment inhibitor and vaccine. Cellular \& Molecular Immunology 19, 1-8, doi: 10.1038/s41423-020-0400-4. On the origin and continuing evolution of SARS-CoV-2. National Science Review nwaa036, https://doi.org/10.1093/nsr/nwaa036. Toll-like receptor 3 signaling via TRIF contributes to a protective innate immune response to severe acute respiratory syndrome coronavirus infection. MBio 6(3), e00638-15. J. 2018. Peptide vaccine against chikungunya virus: immuno-informatics combined with molecular docking approach. Journal of Translational Medicine 16(1), 298. peptide vaccine design and target site depiction against Middle East Respiratory Medicine 17(1), 362. 
804 Van Gunsteren WF, Billeter SR, Eising AA, Hunenberger PH, Krüger PKHC, Mark AE, Scott 805 WRP, Tironi IG, Hünenberger PH, Huenenberger PH, Krueger P. 1996. The 806 GROMOS96 manual and user guide.

807 Wang N, Rosen O, Wang L, Turner HL, Stevens LJ, Corbett KS, Bowman CA, Pallesen J, 808 Shi W, Zhang Y, Leung K. 2019. Structural Definition of a Neutralization-sensitive

809 Epitope on the MERS-CoV S1-NTD. Cell Reports 28(13), 3395-405.

810

Watanabe Y, Allen JD, Wrapp D, McLellan JS, Crispin M. 2020. Site-specific glycan analysis of the SARS-CoV-2 spike. Science eabb9983, doi: 10.1126/science.abb9983.

812 Waterhouse A, Bertoni M, Bienert S, Studer G, Tauriello G, Gumienny R, Heer FT, de Beer TAP, Rempfer C, Bordoli L, Lepore R. 2018. "SWISS-MODEL: homology modelling of protein structures and complexes." Nucleic Acids Research 46(W1), W296-W303.

Waugh DS. 2011. An overview of enzymatic reagents for the removal of affinity tags. Protein Expression and Purification 80(2), 283-293. (WHO).

819 Wolfert MA., Boons GJ. 2013. Adaptive immune activation: glycosylation does matter. Nature Chemical Biology 9(12), 776.

821 Wrapp D, Wang N, Corbett KS, Goldsmith JA, Hsieh CL, Abiona O, Graham BS, McLellan 822 JS. 2020. Cryo-EM structure of the 2019-nCoV spike in the prefusion conformation. Science 367(6483), 1260-1263, doi: 10.1126/science.abb2507. ML. 2020."A new coronavirus associated with human respiratory disease in China." Nature 1-5. 
827 Xue LC, Rodrigues JP, Kastritis PL, Bonvin AM, Vangone A. 2016. "PRODIGY: a web 828 server for predicting the binding affinity of protein-protein complexes." 829 Bioinformatics 32(23), 3676-3678.

830 Yazdani Z, Rafiei A, Yazdani M, Valadan R. 2020. Design an efficient multi-epitope peptide 831 vaccine candidate against SARS-CoV-2: An in silico analysis. bioRxiv doi: 832 https://doi.org/10.1101/2020.04.20.051557.

833 Yong CY, Ong HK, Yeap SK, Ho KL, Tan WS. 2019. Recent Advances in the Vaccine 834 Development Against Middle East Respiratory Syndrome-Coronavirus. Frontiers in $835 \quad$ Microbiololgy 10, 1781.

836 Zander RA, Vijay R, Pack AD, Guthmiller JJ, Graham AC, Lindner SE, Vaughan AM, Kappe 837 SH, Butler NS. 2017. Th1-like plasmodium-specific memory CD4+ T cells support 838 humoral immunity. Cell Reports 21(7), 1839-1852.

839 Zhou D, Qi R, Zhang W, Tian X, Peng C. 2020b. Identification of 22 N-glycosites on Spike 840 Glycoprotein of SARS-CoV-2 and Accessible Surface Glycopeptide Motifs: Implications on Vaccination and Antibody Therapeutics. Preprints, 2020020381, doi: 10.20944/preprints202002.0381.v2.

844 neutralization epitope on the N-terminal domain of MERS-CoV spike glycoprotein. Nature Communications 10, 3068.

846 Zhou P, Yang XL, Wang XG, Hu B, Zhang L. et al. 2020a. Discovery of a novel coronavirus 847 associated with the recent pneumonia outbreak in humans and its potential bat origin. Nature doi: 10.1038/s41586-020-2012-7. 
Figure 1

Fig. 1

The three-dimensional (3D) structure of the $\mathrm{N}$-terminal domains (NTDs) and receptor binding domains (RBDs) of the spike (S) proteins of SARS-CoV-2 (surface view). The orange, cyan, and yellow colored regions represent the potential antigenic domains predicted by the IEDB analysis resource ElliPro analysis.

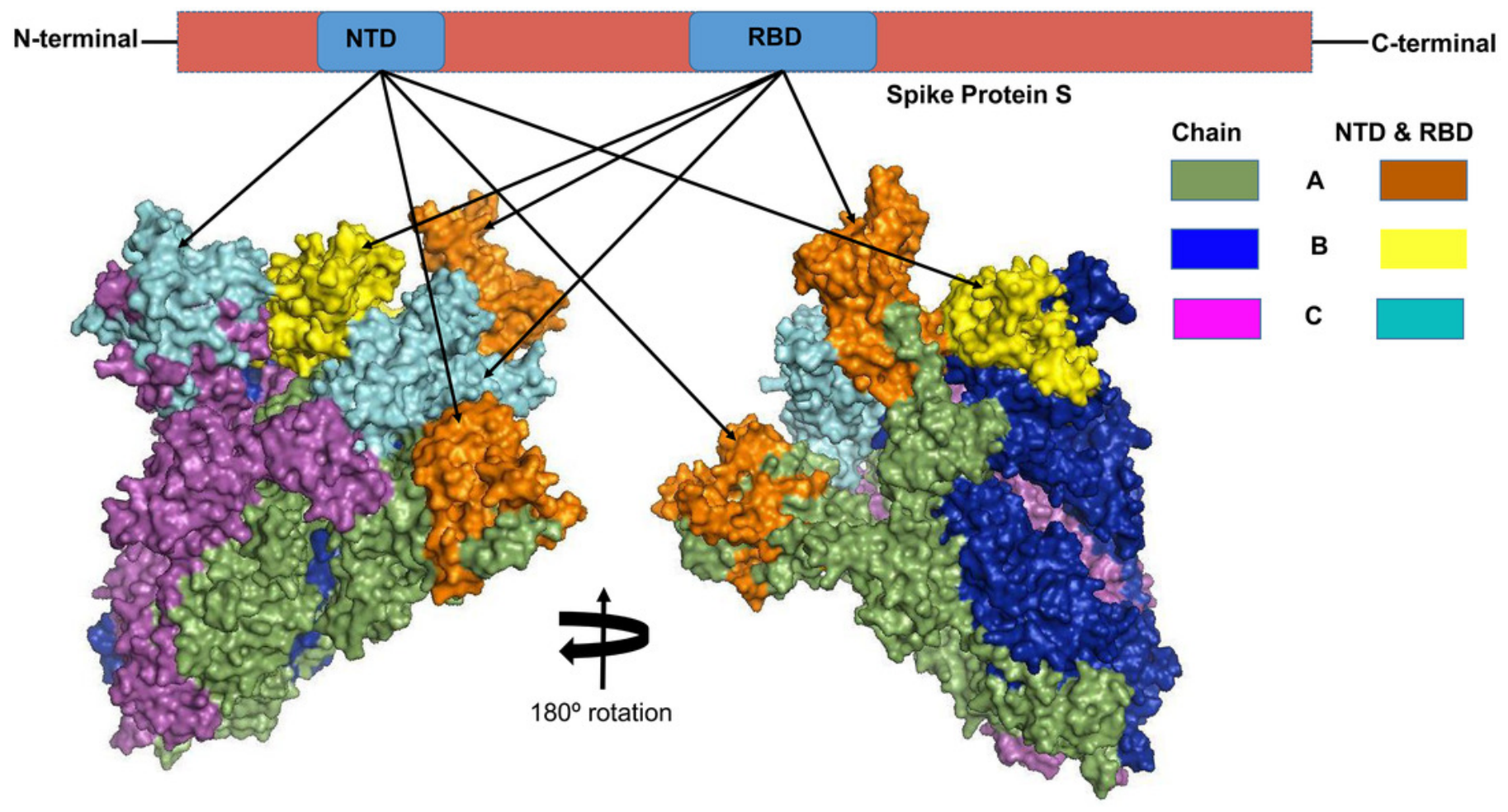


Figure 2

Fig. 2

Predicted B-cell epitopes using BepiPred-2.0 epitope predictor in IEDB-analysis resource web-based repository. Yellow areas above threshold (red line) are proposed to be a part of B cell epitopes in (a) RBD and (b) NTD regions of S protein, (c) envelop (E) and (d) membrane (M) proteins of SARS-CoV-2.
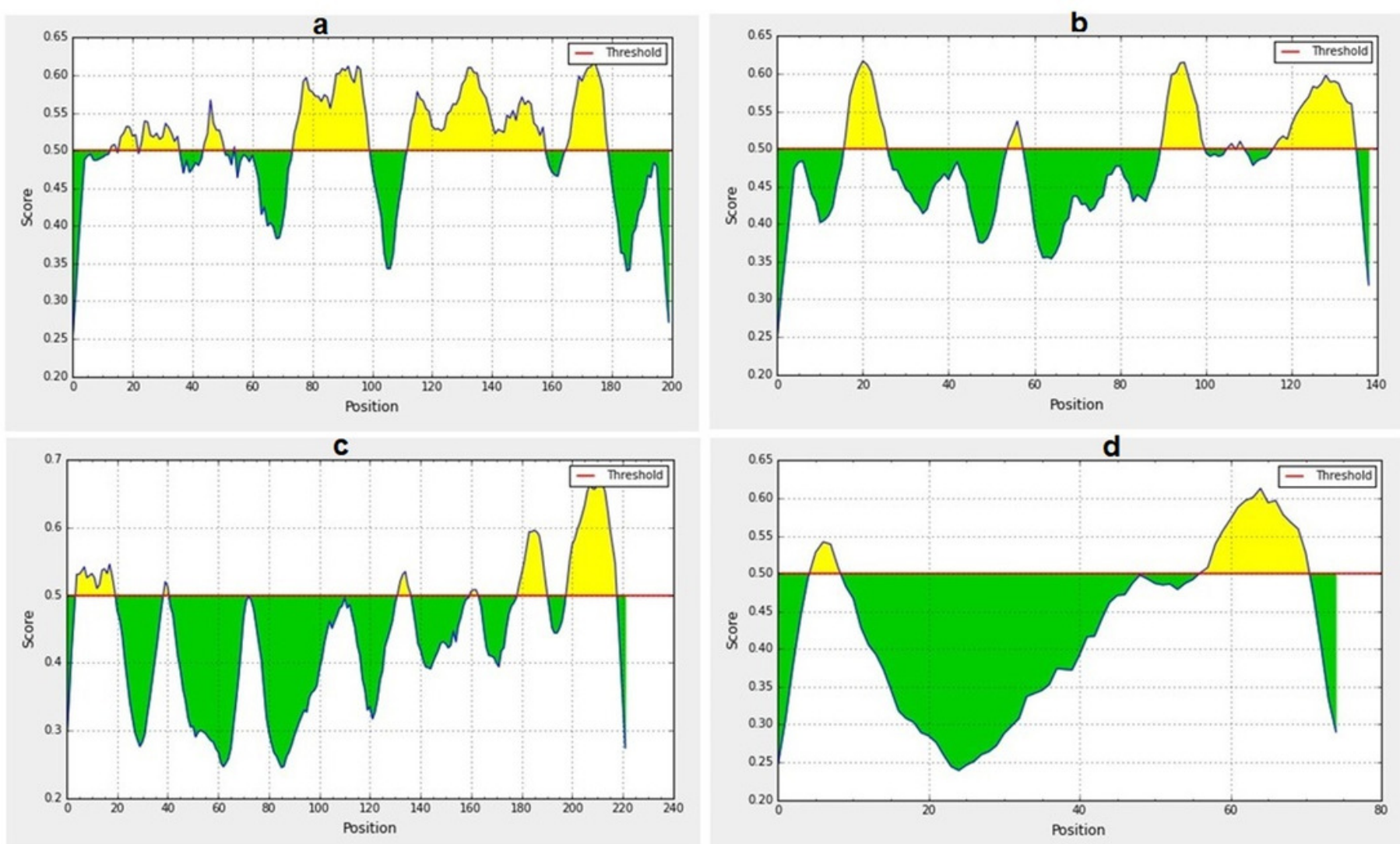


\section{Figure 3}

Fig. 3

\section{Design, construction and structural validation of multi-epitope vaccine candidate}

(CoV-RMEN) for SARS-CoV-2. (a) Structural domains and epitopes rearrangement of CoV-

RMEN, (b) secondary structure of CoV-RMEN as analyzed through CFSSP:Chou and Fasman

secondary structure prediction server , (c) final tertiary structure of CoV-RMEN (surface view) obtained from homology modelling on Phyre2 in which domains and epitopes are represented in different colors (PADRE-smudge; membrane B-cell epitope, MBE-magenta; $\mathrm{N}$ terminal domain, NTD-orange; receptor-binding domain, RBD-cyan; envelop B-cell epitope, EBE-blue; invasin-yellow), (d) validation of the refined model with Ramachandran plot analysis showing $94.7 \%, 4.8 \%$ and $0.5 \%$ of protein residues in favored, allowed, and disallowed (outlier) regions respectively, (e) ProSA-web, giving a Z-score of -6.17 , and (f) the finally predicted primary structure of the CoV-RMEN. 


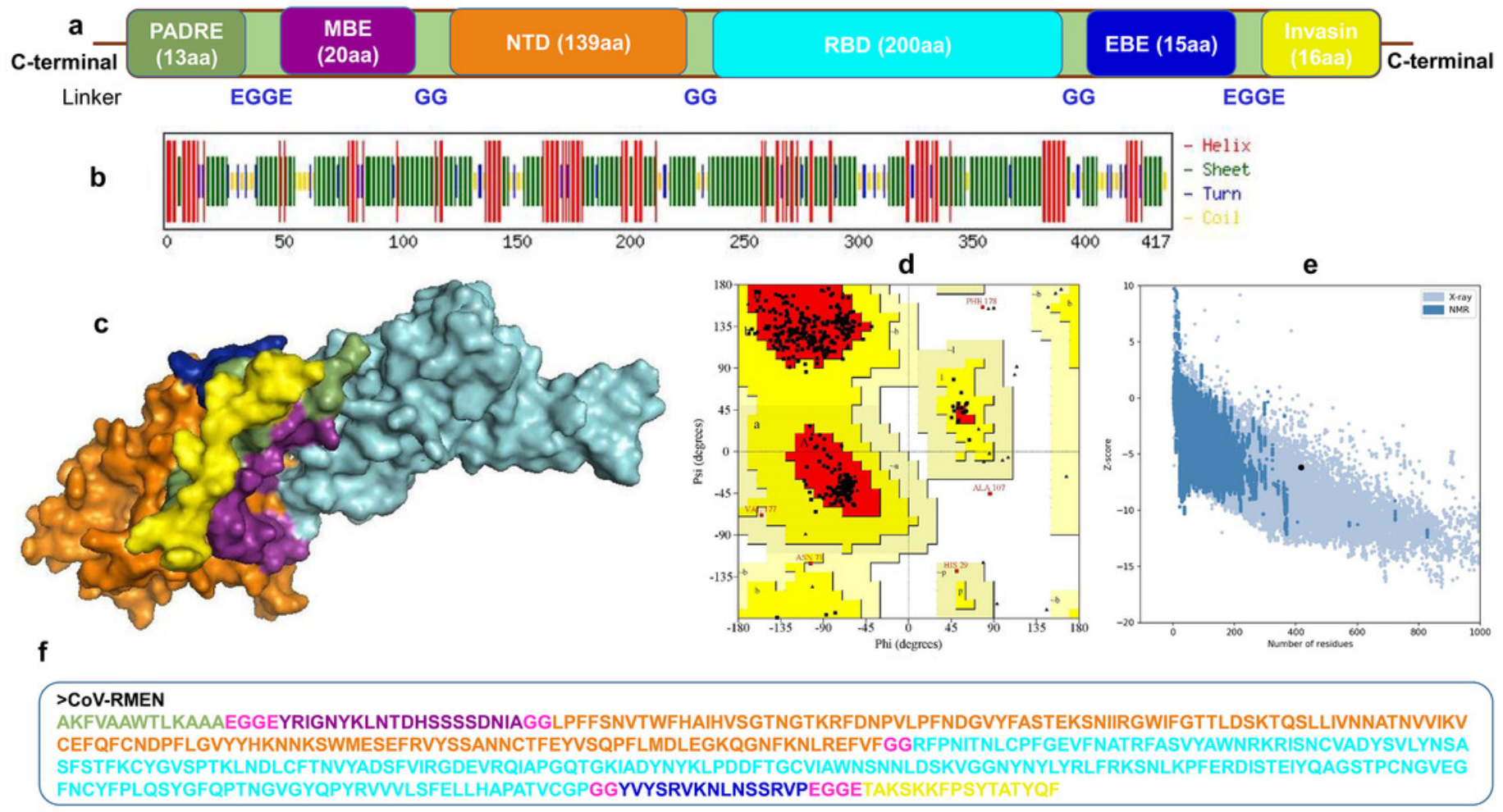




\section{Figure 4}

Fig. 4

\section{Molecular docking of top five MHC-I and MHC-II epitopes of RBD and NTD domains} with respect to HLA allele binders. Figures (a-e) and (k-0) represent the top five MHC-I epitopes of RBD and NTD domains, respectively. Figures $(f-j)$ and $(p-t)$ represent the top five MHC-II epitopes binds of the same domains binds to their respective HLA alleles. The proteinpeptide docking was performed in GalaxyWEB-GalaxyPepDock-server followed by the refinement using GalaxyRefineComplex and free energy $(\Delta G)$ of each complex was determined in PRODIGY server. Ribbon structures represent HLA alleles and stick structures represent the respective epitopes. Light color represents the templates to which the alleles and epitopes structures were built. Further information on molecular docking analysis is also available in Supplemental Data 1. 


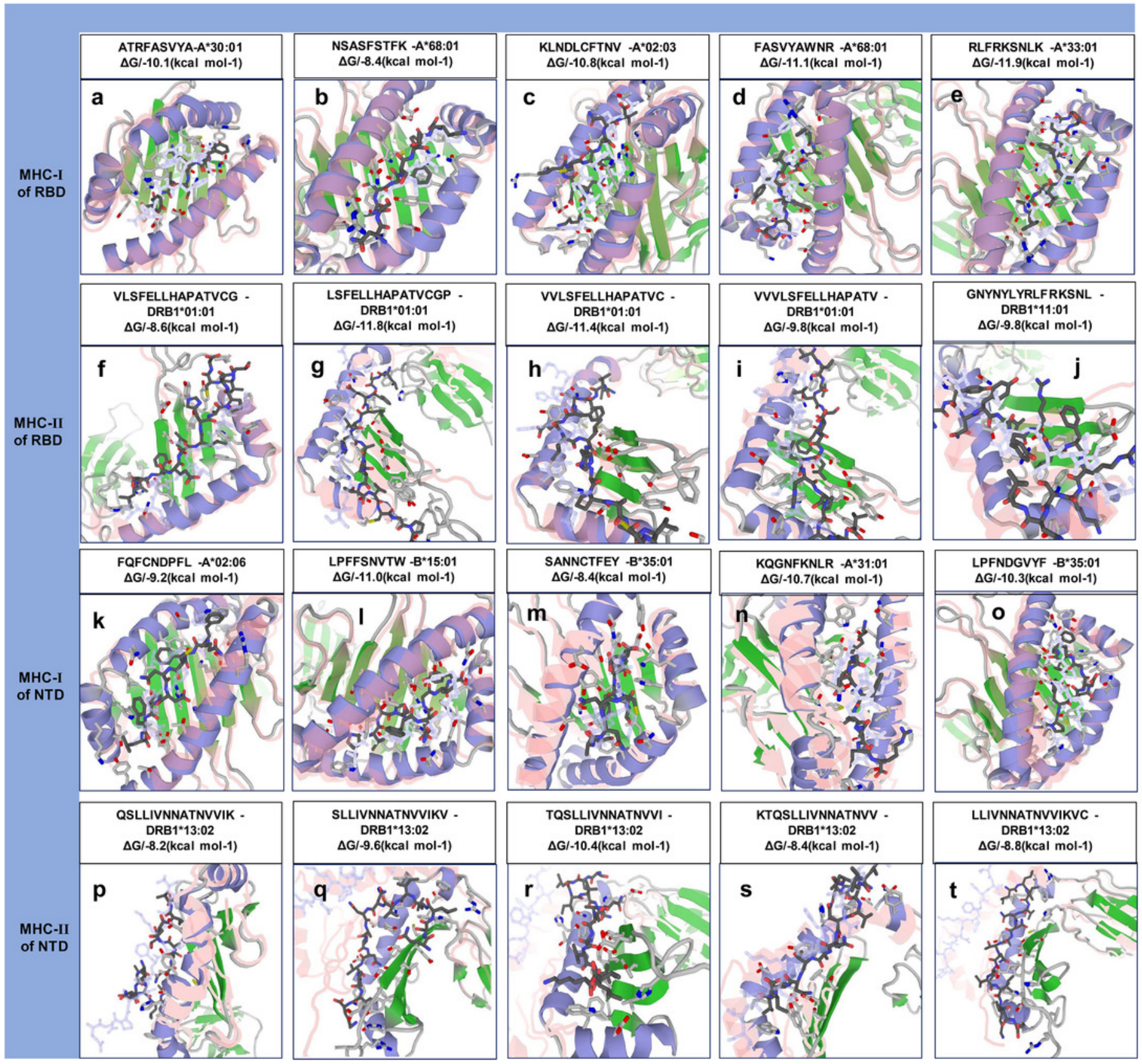




\section{Figure 5}

Fig. 5

Molecular docking and dynamics of CoV-RMEN vaccine with immune receptors

(TLR2, TLR3 and TLR4). Docked complexes for (a) CoV-RMEN and TLR2, (b) CoV-RMEN and

TLR3, and (c) CoV-RMEN and TLR4. Magnified interfaces of the respective complexes are

figured to (d), (e) and (f) respectively. Active residues of CoV-RMEN colored magenta, and of TLRs colored orange with stick view. $\Delta G$ represents the binding affinity of the complexes. Molecular dynamics simulation study of (g) CoV-RMEN and TLR2, (h) CoV-RMEN and TLR3, and (i) CoV-RMEN and TLR4 complexes across the time window of 100 ps. The reasonably invariable RMSD value indicates a stable complex formation. 

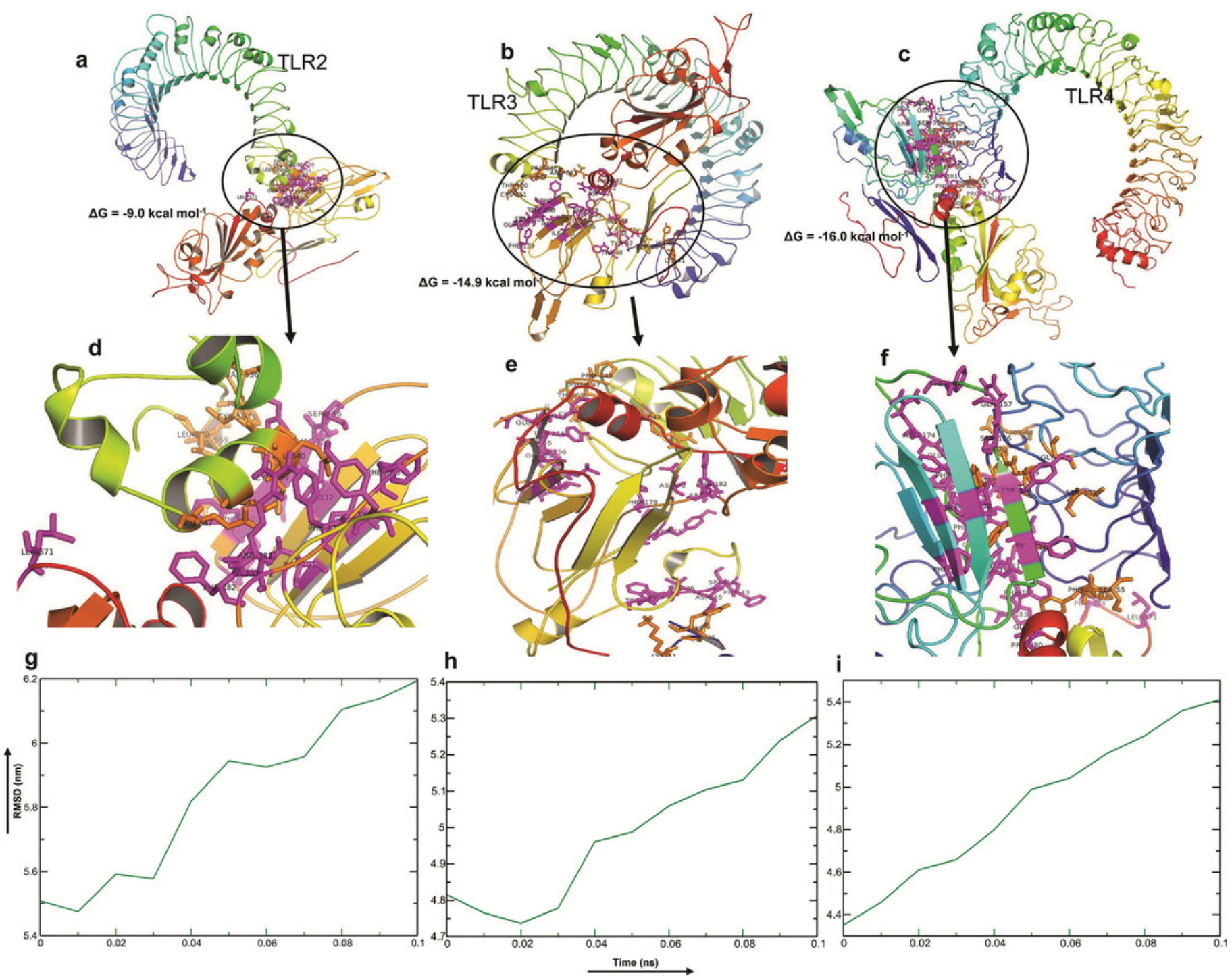


\section{Figure 6}

Fig. 6

\section{C-ImmSim presentation of an in silico immune simulation with the chimeric}

peptide. (a) The immunoglobulins and the immunocomplex response to antigen (CoV-RMEN) inoculations (black vertical lines); specific subclasses are indicated as colored peaks, (b) concentration of cytokines and interleukins, and inset plot shows danger signal together with leukocyte growth factor IL-2, (c) B-cell populations after three injections, (d) evolution of B cell, (e) T-helper cell populations per state after injections, and (f) evolution of T-helper cell classes with the course of vaccination. 

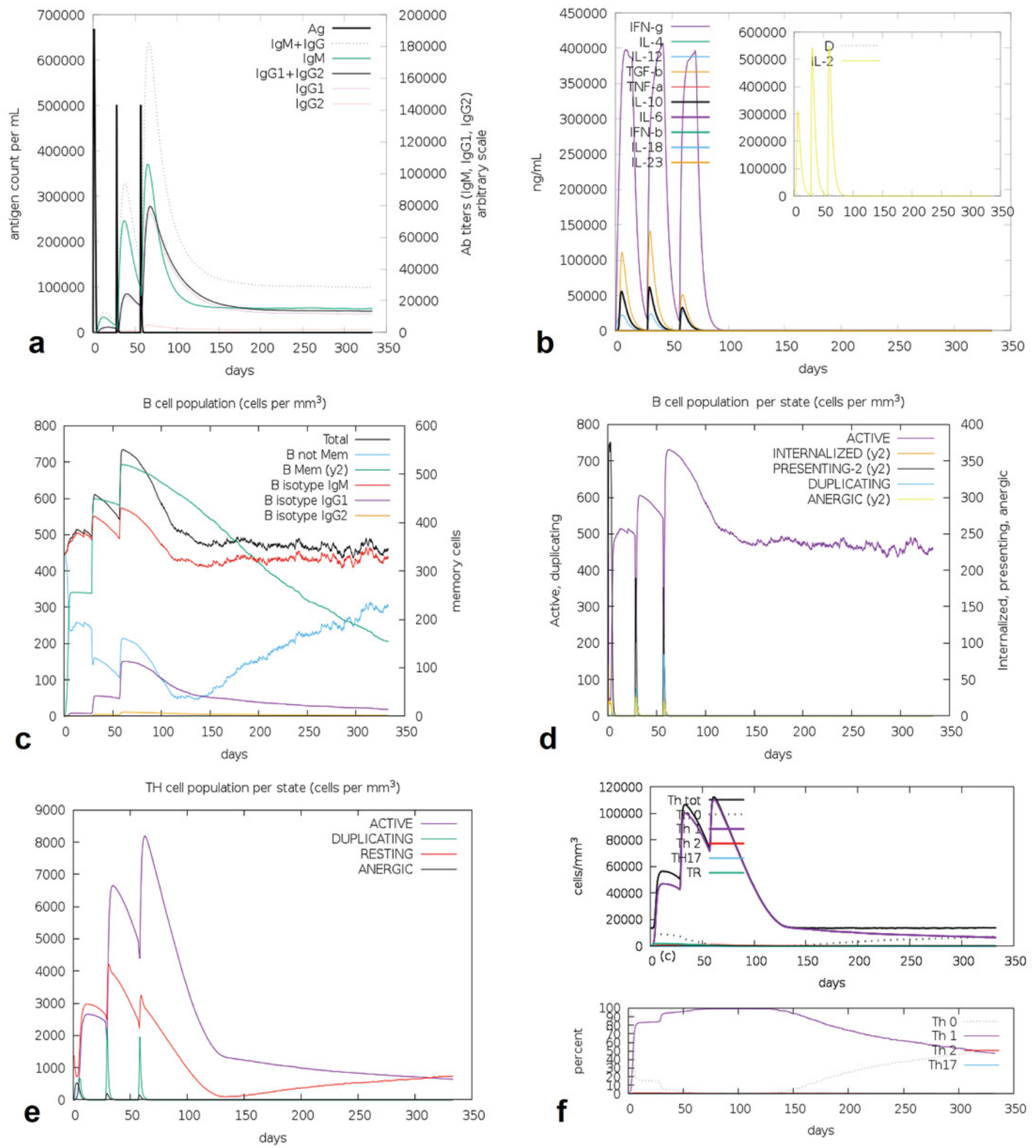


\section{Figure 7}

Fig. 7

\section{Population coverage of the selected T-cell epitopes and their respective HLA}

alleles. The circular plot illustrates the relative abundance of the top 70 geographic regions and ethnic groups for selected CTL and HTL epitopes, which were used to construct the vaccine and their corresponding MHC HLA alleles were obtained for population coverage analysis both individually (either MHC-I or MHC-II) and in combination (MHC-I and MHC-II). (a) Population coverage of top seventy geographical regions out of 123 regions. (b) Population coverage of top seventy ethnic groups selected from 146 ethnic groups. Regions and ethnic groups in the respective MHC-I and MHC-II epitopes are represented by different colored ribbons, and the inner blue bars indicate their respective relative coverages. Further information on population coverage analysis is also available in Supplemental Data 1.
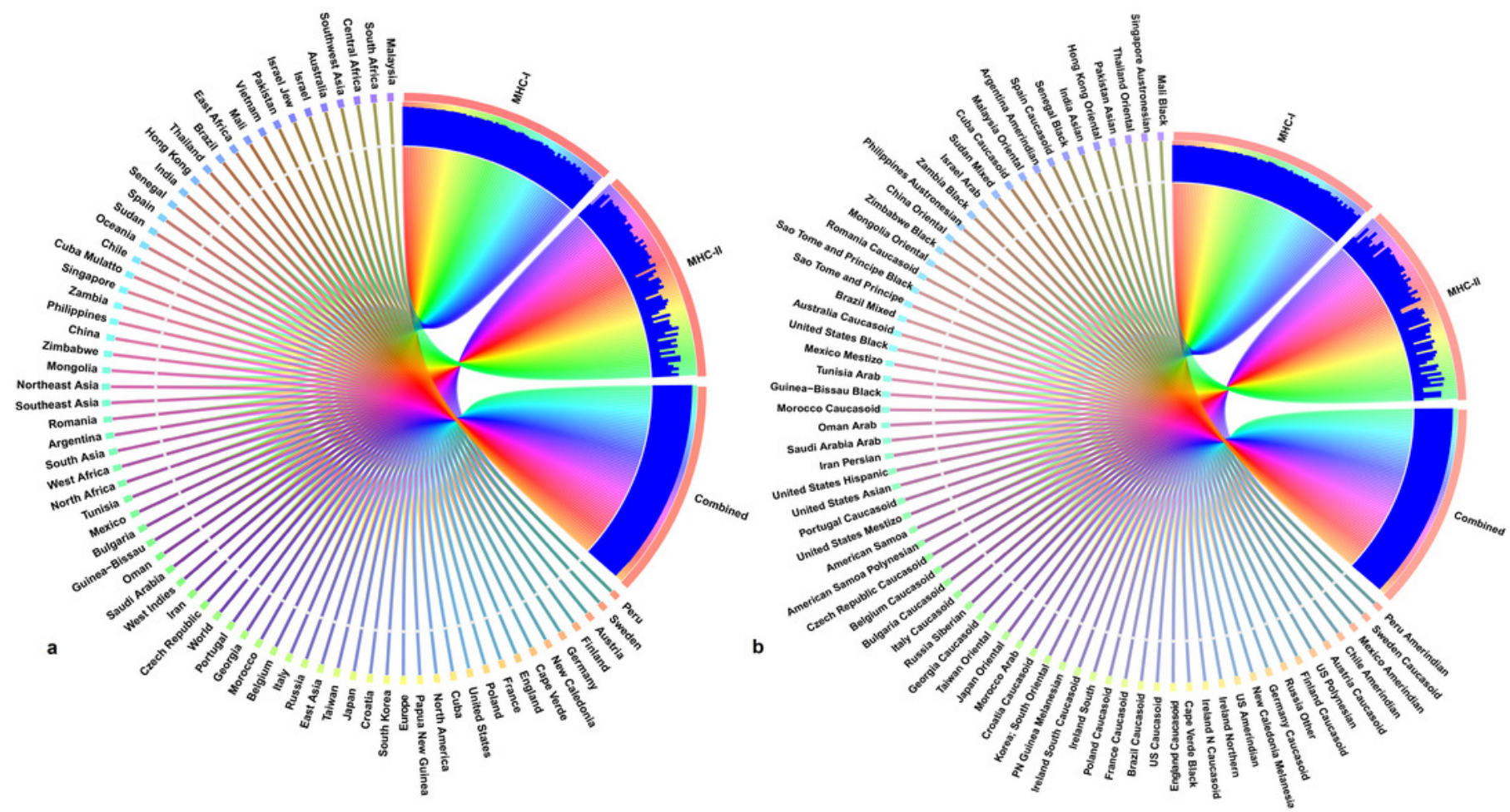
Figure 8

Fig. 8

\section{Codon optimization and mRNA structure of CoV-RMEN gene for expression in $E$.}

coli (a) GC curve (average GC content: $50.26 \%$ ) of the optimized CoV-RMEN gene, (b) percentage distribution of codons in computed codon quality groups, (c) relative distribution of codon usage frequency along the gene sequence to be expressed in E. coli, and codon adaptation index (CAl) was found to be 0.87 for the desired gene, (d) secondary structure and stability of corresponding mRNA, and (e) resolved view of the start region in the mRNA structure of CoV-RMEN.
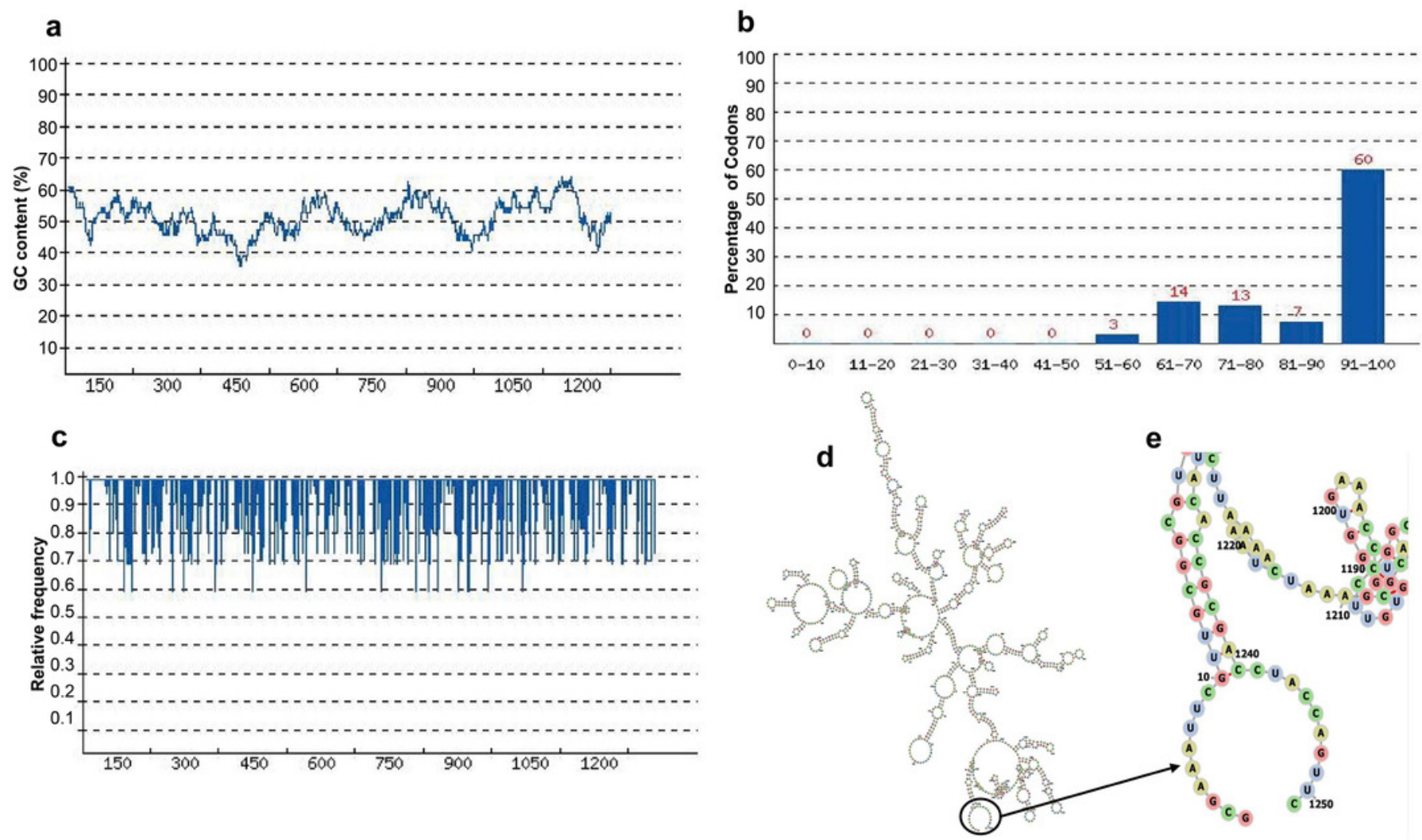
Figure 9

Fig. 9

In silico fusion cloning of the CoV-RMEN. The final vaccine candidate sequence was inserted into the pETite expression vector where the red part represents the gene coding for the predicted vaccine, and the black circle represents the vector backbone. The six His-tag and SUMU-tag are located at the Carboxy-terminal end.

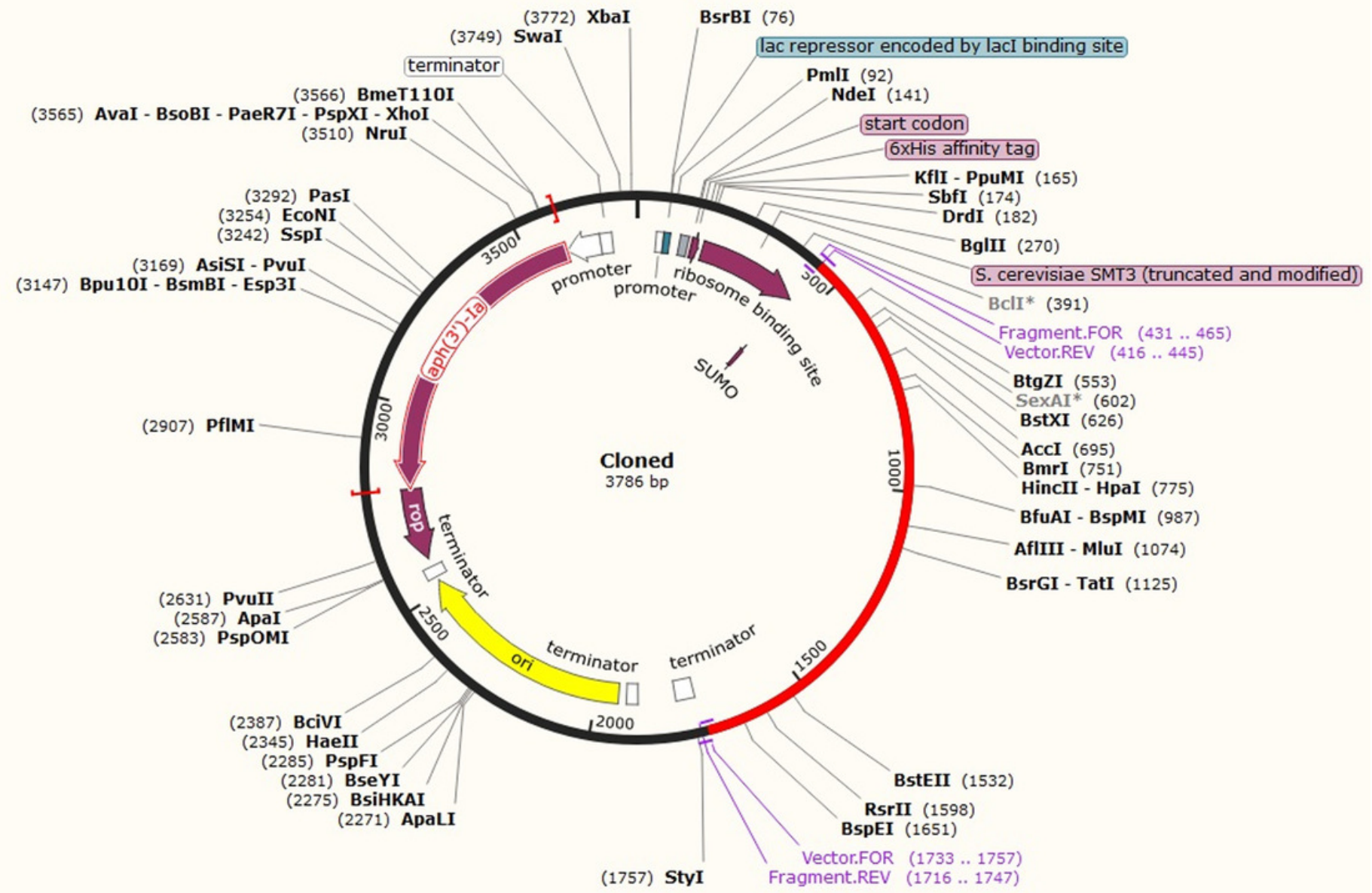




\section{Table $\mathbf{1}$ (on next page)}

Table 1

Linear epitopes present on spike (S) glycoprotein surface predicted through ElliPro in IEDBanalysis resource based upon solvent-accessibility and flexibility are shown with their antigenicity scores. The highlighted green coloured regions were the potential antigenic domains while the yellow coloured region represents the trans-membrane domain of the $S$ protein. 


\section{Epitope-based chimeric peptide vaccine design against S, M, and E proteins of SARS-CoV-}

\section{2 etiologic agent of global pandemic COVID-19: an in silico approach}

3 M. Shaminur Rahman, M. Nazmul Hoque, M. Rafiul Islam, Salma Akter, A.S.M. Rubayet- Ul-

4 Alam, Mohammad Anwar Siddique, Otun Saha, Md. Mizanur Rahaman, Munawar Sultana, Keith

5 A. Crandall, M. Anwar Hossain

6 Table 1. Linear epitopes present on spike (S) glycoprotein surface predicted through ElliPro in

7 IEDB-analysis resource based upon solvent-accessibility and flexibility are shown with their

8 antigenicity scores. The highlighted green coloured regions were the potential antigenic domains

9 while the yellow coloured region represents the trans-membrane domain of the S protein.

10

\begin{tabular}{|c|c|c|c|c|c|c|}
\hline No. & Chain & Start & End & $\begin{array}{c}\text { Peptide } \\
\end{array}$ & Residues & Score \\
\hline 1 & \multirow{15}{*}{ Tram } & 395 & 514 & $\begin{array}{c}\text { VYADSFVIRGDEVRQIAPGQTGKIADYNYKLPDDFTGCVIAWNSNNLDSKVGGNYNYLYRLFRKSNLKPF } \\
\text { ERDISTEIYQAGSTPCNGVEGFNCYFPLQSYGFQPTNGVGYPYRVVLS }\end{array}$ & 120 & 0.837 \\
\hline 2 & & 58 & 194 & $\begin{array}{l}\text { FFSNVTWFHAIHVSGTNGTKRFDNPVLPFNDGVYFASTEKSNIIRGWIFGTTLDSKTQSLLIVNNATNVVIK } \\
\text { VCEFQFCNDPFLGVYYHKNNKSWMESEFRVYSSANNCTFEYVSQPFLMDLEGKQGNFKNLREFFF }\end{array}$ & 137 & 0.835 \\
\hline 3 & & 1067 & 1146 & $\begin{array}{l}\begin{array}{l}\text { YVPAQEKNFTTAPAICHDGKAHFPREGVFVSNGTHWFVTQRNFYEPQIITTDNTFVSGNCDVVIGIVNNT } \\
\text { VYDPLQPELD }\end{array} \\
\end{array}$ & 80 & 0.83 \\
\hline 4 & & 201 & 270 & FKIYSKHTPINLVRDLPQGFSALEPLVDLPIGINITRFQTLLALHRSYLTPGDSSSGWTAGAAAYYVGYL & 70 & 0.76 \\
\hline 5 & & 331 & 381 & NITNLCPFGEVFNATRFASVYAWNRKRISNCVADYSVLYNSASFSTFKCYG & 51 & 0.706 \\
\hline 6 & & 700 & 720 & GAENSVAYSNNSIAIPTNFTI & 21 & 0.668 \\
\hline 7 & & 27 & 35 & AYTNSFTRG & 9 & 0.66 \\
\hline 8 & & 909 & 936 & IGVTQNVLYENQKLIANQFNSAIGKIQD & 28 & 0.633 \\
\hline 9 & & 789 & 813 & YKTPPIKDFGGFNFSQILPDPSKPS & 25 & 0.6 \\
\hline 10 & & 623 & 642 & AIHADQLTPTWRVYSTGSNV & 20 & 0.598 \\
\hline 11 & & 891 & 907 & GAALQIPFAMQMAYRFN & 17 & 0.591 \\
\hline 12 & & 579 & 583 & PQTLE & 5 & 0.551 \\
\hline 13 & & 687 & 692 & VASQSI & 6 & 0.55 \\
\hline 14 & & 653 & 659 & AEHVNNS & 7 & 0.539 \\
\hline 15 & & 679 & 684 & NSPRRA & 6 & 0.521 \\
\hline 16 & \multirow{18}{*}{ B } & 1067 & 1146 & $\begin{array}{l}\text { YVPAQEKNFTTAPAICHDGKAHFPREGVFVSNGTHWFVTQRNFYEPQIITTDNTFVSGNCDVVIGIVNNT } \\
\text { VYDPLQPELD }\end{array}$ & 80 & 0.826 \\
\hline 17 & & 89 & 194 & $\begin{array}{c}\text { GVYFASTEKSNIIRGWIFGTTLDSKTQSLLIVNNATNVVIKVCEFQFCNDPFLGVYYHKNNKSWMESEFRV } \\
\text { YSSANNCTFEYVSQPFLMDLEGKQGNFKNLREFVF }\end{array}$ & 106 & 0.816 \\
\hline 18 & & 58 & 87 & FFSNVTWFHAIHVSGTNGTKRFDNPVLPFN & 30 & 0.81 \\
\hline 19 & & 203 & 270 & IYSKHTPINLVRDLPQGFSALEPLVDLPIGINITRFQTLLALHRSYLTPGDSSSGWTAGAAYYYVGYL & 68 & 0.748 \\
\hline 20 & & 465 & 509 & ERDISTEIYQAGSTPCNGVEGFNCYFPLQSYGFQPTNGVGYQPYR & 45 & 0.727 \\
\hline 21 & & 436 & 458 & WNSNNLDSKKGGNYNYLYRLFRK & 23 & 0.672 \\
\hline 22 & & 700 & 720 & GAENSVAYSNNSIAIPTNFTI & 21 & 0.671 \\
\hline 23 & & 27 & 35 & AYTNSFTRG & 9 & 0.666 \\
\hline 24 & & 909 & 9036 & IGVTQNVLYENQKLIANQFNSAIGKIQD & 28 & 0.641 \\
\hline 25 & & 624 & 643 & IHADQLTPTWRVYSTGSNVF & 20 & 0.617 \\
\hline 26 & & 328 & 365 & RFPNITNLCPFGEVFNATRFASVYAWNRKRISNCVADY & 38 & 0.608 \\
\hline 27 & & 891 & 907 & GAALQIPFAMQMAYRFN & 17 & 0.602 \\
\hline 28 & & 577 & 583 & RDPQTLE & 7 & 0.598 \\
\hline 29 & & 790 & 817 & KTPPIKDFGGFNFSQILPDPSKPSKRSF & 28 & 0.595 \\
\hline 30 & & 673 & 693 & SYQTQTNSPRRARSVASQSII & 21 & 0.567 \\
\hline 31 & & 526 & 537 & GPKKSTNLVKNK & 12 & 0.553 \\
\hline 32 & & 653 & 661 & AEHVNNSYE & 9 & 0.548 \\
\hline 33 & & 554 & 563 & $\begin{array}{ll}\text { ESNKKFLPFQ } \\
\end{array}$ & 10 & 0.52 \\
\hline 34 & \multirow{14}{*}{ C } & 56 & 194 & $\begin{array}{l}\text { LPFFSNVTWFHAIHVSGTNGTKRFDNPVLPFNDGVYFASTEKSNIIRGWIFGTTLDSKTQSLLIVNNATNV } \\
\text { VIKVCEFQFCNDPFLGVYYHKNNKSWMESEFRVYSSANNCTFEYVSQPFLMDLEGKQGNFKNLREFVF }\end{array}$ & 139 & 0.84 \\
\hline 35 & & 1067 & 1146 & 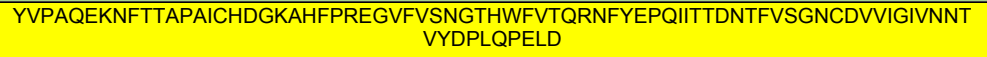 & 80 & 0.822 \\
\hline 36 & & 201 & 270 & FKIYSKHTPINLVRDLPQGFSALEPLVDLPIGINITRFQTLLALHRSYLTPGDSSSGWTAGAAAYYVGYL & 70 & 0.77 \\
\hline 37 & & 27 & 35 & $\begin{array}{c}\text { AYTNSFTRG } \\
\end{array}$ & 9 & 0.676 \\
\hline 38 & & 465 & 509 & ERDISTEIYQAGSTPCNGVEGFNCYFPLQSYGFQPTNGVGYQPYR & 45 & 0.675 \\
\hline 39 & & 700 & 720 & GAENSVAYSNNSIAIPTNFTI & 21 & 0.658 \\
\hline 40 & & 909 & 936 & IGVTQNVLYENQKLIANQFNSAIGKIQD & 28 & 0.633 \\
\hline 41 & & 437 & 458 & NSNNLDSKVGGNYNYLYRLFRK & 22 & 0.629 \\
\hline 42 & & 673 & 684 & SYQTQTNSPRRA & 12 & 0.619 \\
\hline 43 & & 790 & 817 & KTPPIKDFGGFNFSQILPDPSKPSKRSF & 28 & 0.602 \\
\hline 44 & & 891 & 907 & GAALQIPFAMQMAYRFN & 17 & 0.597 \\
\hline 45 & & 578 & 583 & DPQTLE & 6 & 0.589 \\
\hline 46 & & 620 & 631 & VPVAIHADQLTP & 12 & 0.579 \\
\hline 47 & & 329 & 362 & $\begin{array}{l}\text { FPNITNLCPFGEVFNATRFASVYAWNRKRISNCV } \\
\end{array}$ & 34 & 0.571 \\
\hline
\end{tabular}


11

\begin{tabular}{|c|c|c|c|c|c|}
\hline 48 & 687 & 692 & VASQSI & 6 & 0.566 \\
\hline 49 & 835 & 845 & KQYGDCLGDIA & 11 & 0.567 \\
\hline 50 & 653 & 659 & AEHVNNS & 7 & 0.559 \\
\hline 51 & 527 & 536 & PKKSTNLVKN & 10 & 0.546 \\
\hline 52 & 635 & 642 & VYSTGSNV & 8 & 0.51 \\
\hline
\end{tabular}




\section{Table 2 (on next page)}

Table 2

B-cell epitopes predicted using Bepipred linear epitope prediction 2.0 in IEDB analysis resource web-server along with their start and end positions, average score, and Vaxijen 2.0 determined antigenicity scores. 
1 Table 2: B-cell epitopes predicted using Bepipred linear epitope prediction 2.0 in IEDB analysis 2 resource web-server along with their start and end positions, average score, and VaxiJen 2.0

3 determined antigenicity scores.

\begin{tabular}{|c|c|c|c|c|}
\hline $\begin{array}{l}\text { Domain/pro } \\
\text { teins }\end{array}$ & Position & Sequences & $\begin{array}{c}\text { Average } \\
\text { Score }\end{array}$ & Antigenicity \\
\hline \multirow{8}{*}{ RBD } & $341-342$ & VF & 0.502 & - \\
\hline & 344-349 & ATRFAS & 0.520 & -0.151 \\
\hline & $351-363$ & YAWNRKRISNCVA & 0.522 & 0.394 \\
\hline & $372-378$ & ASFSTFK & 0.527 & 0.087 \\
\hline & 382 & $\mathrm{~V}$ & 0.464 & - \\
\hline & $402-427$ & IRGDEVRQIAPGQTGKIADYNYKLPD & 0.575 & 0.932 \\
\hline & $440-485$ & $\begin{array}{c}\text { NLDSKVGGNYNYLYRLFRKSNLKPFER } \\
\text { DISTEIYQAGSTPCNGVEG }\end{array}$ & 0.554 & 0.210 \\
\hline & $493-516$ & QSYGFQPTNGVGYQ & 0.535 & 0.670 \\
\hline \multirow{6}{*}{ NTD } & $72-81$ & GTNGTKRFDN & 0.573 & 0.667 \\
\hline & $110-113$ & LDSK & 0.511 & - \\
\hline & $146-155$ & HKNNKSWMES & 0.573 & 0.174 \\
\hline & $161-162$ & $\mathrm{SS}$ & 0.503 & - \\
\hline & 164 & $\mathrm{~N}$ & 0.499 & - \\
\hline & $172-191$ & SQPFLMDLEGKQGNFKNLRE & 0.553 & 0.749 \\
\hline MBE & $199-218$ & YRIGNYKLNTDHSSSSDNIA & 0.614 & 0.222 \\
\hline EBE & $57-71$ & YVYSRVKNLNSSRVP & 0.565 & 0.449 \\
\hline
\end{tabular}




\section{Table 3(on next page)}

Table 3

Active interface amino acid residues and binding scores among Toll Like Receptors (TLRs) and the constructed vaccine CoV-RMEN. 
1 Epitope-based chimeric peptide vaccine design against $\mathrm{S}, \mathrm{M}$, and $\mathrm{E}$ proteins of $\mathrm{SARS}-\mathrm{CoV}$ -

2

3 M. Shaminur Rahman, M. Nazmul Hoque, M. Rafiul Islam, Salma Akter, A.S.M. Rubayet- Ul-

4 Alam, Mohammad Anwar Siddique, Otun Saha, Md. Mizanur Rahaman, Munawar Sultana, Keith

5 A. Crandall, M. Anwar Hossain

6

7 Table 3. Active interface amino acid residues and binding scores among Toll Like Receptors 8 (TLRs) and the constructed vaccine CoV-RMEN.

\begin{tabular}{|c|c|c|c|c|}
\hline \multicolumn{2}{|c|}{ Active residues of TLRs } & Active residues of $\mathrm{CoV}$-RMEN & $\begin{array}{c}\text { HADDOCK } \\
\text { score }\end{array}$ & $\Delta G\left(\right.$ kcal mol $\left.^{-1}\right)$ \\
\hline TLR-2 & $\begin{array}{llll}\text { V536, } & \text { C537, } & \text { S538, } & \text { C539, } \\
\text { E540, S543, } & \text { E547, } & \text { P567, } \\
\text { R569, L570 } & & \end{array}$ & $\begin{array}{l}\text { D72, Y75, L101, I103, I112, C150, } \\
\text { F152, E153, Y154, V155, S156, } \\
\text { F176, F178, R181, F182, L371 }\end{array}$ & -30.4 & -9.0 \\
\hline TLR-3 & $\begin{array}{l}\text { D36, H39, K41, R643, F644, } \\
\text { P646, F647, T650, C651, } \\
\text { E652, S653, I654, W656, } \\
\text { F657, V658, N659, W660, } \\
\text { I661, N662, E663 }\end{array}$ & $\begin{array}{l}\text { F43, S44, N45, V46, T47, W48, } \\
\text { D72, Y75, F76, L101, I103, I112, } \\
\text { F152, E153, Y154, V155, S156, } \\
\text { Q157, F159, F178, R181, F182, } \\
\text { L371 }\end{array}$ & -47.2 & -14.9 \\
\hline TLR-4 & $\begin{array}{l}\text { P53, F54, S55, H68, G70, } \\
\text { Y72, S73, F75, S76, Q99, } \\
\text { S102, G124, }\end{array}$ & $\begin{array}{l}\text { G39, L40, D72, Y75, F76, F90, } \\
\text { L101, I103, I112, F152, Y154, } \\
\text { S156, Q157, F159, R174, E175, } \\
\text { F176, F178, R181, F182, P183, } \\
\text { L371, P374, P380, G381 }\end{array}$ & -52.1 & -16.0 \\
\hline
\end{tabular}

9

10 